\title{
„Fördern und Fordern“ - Aktivierung am Arbeitsmarkt in Deutschland und Großbritannien
}

\author{
Bernd Schulte
}

I. Vorbemerkung: „Vom Nutzen und Frommen“ des Sozialrechtsvergleichs

II. Fürsorge/,SSocial Assistance“ in der Europäischen Union 275

III. Die Entwicklung des sozialen Schutzes in Deutschland und Großbritannien 280

1. 19. Jahrhundert: Traditionelle nationale „Pfade“ der sozialpolitischen Entwicklung

2. 20. Jahrhundert: Entstehung und Entwicklung von Welfarestate und Sozialstaat

IV. „Fürsorge“/,Social Assistance“ als Teil der sozialen Sicherheit

V. Die Rolle des Rechts und der Gerichtsbarkeit bei der Verwirklichung sozialer Rechte

VI. Reform der Arbeitsförderung in Großbritannien und in Deutschland: „Harrods“ und „Hartz“

1. Europarechtliche und -politische Vorgaben 296

2. Aktivierungspolitik in Großbritannien und Deutschland in Stichworten 297

a) Großbritannien: Die konservative Ära und Labour's Third Way

b) Deutschland „Fördern und Fordern“: „Moderne Dienstleistungen am Arbeitsmarkt" als Teil der „Agenda 2010“

c) Würdigung

VII. Großbritannien und Deutschland - Gemeinsamkeiten und Unterschiede in Stichworten

„The history of anti-poverty schemes is an indictment of the human capacity to overcomplicate and to condemn. In the early years of the $21^{\text {st }}$ century, even the richest countries in the world have levels of poverty that few social thinkers would have anticipated a century ago if told what their countries' per capita incomes would be today. The saying that "the poor are always with us" is only true because politicians and policymakers choose that this should be the case. And yet the political rhetoric is constant that the eradication of poverty is the primary goal of social and economic policy. (...) According to the modernizers the poor in rich societies are in that state because of cruel combination, of lack of capability and induced dependency, from which they need to be rescued. Therefore, they reason, if we want to help the poor, policy must increase their capabilities and induce the unfortunate to overcome their social exclusion, by guidance, direction and coercion if necessary. " $j$

1 Standing, G., Introduction, in: ders. (Hrsg.), Minimum Income Schemes in Europe, Geneva (International Labour Office) 2008, S. 1. 


\section{Vorbemerkung: „Vom Nutzen und Frommen“ des Sozialrechtsvergleichs}

Dient der Rechtsvergleich dem besseren Erfassen, Verstehen und Bewerten von Recht, ${ }^{2}$ so meint „Recht“" in diesem Zusammenhang vor allem das eigene Recht des Betrachters, lässt doch das Aufsuchen vergleichbarer Probleme und Problemlösungen in verschiedenen Rechtsordnungen gleichsam im Sinne einer „Differentialdiagnose“ sowohl die Originalität der jeweiligen eigenen nationalen Lösung als auch - die jeweilige nationale Rechtsordnung überschreitend - die Struktur des rechtlichen Problems überhaupt und der zu seiner Lösung auch anderswo gefundenen Regelungen schärfer und klarer hervortreten. Der Rechtsvergleich führt dadurch zu einem größeren „Vorrat“ sowohl an vorrechtlichen Überlegungen zur Problemanalyse als auch an rechtlichen Erwägungen zu Auslegung, Bewertung und letztlich auch rechtspolitischer Gestaltung des jeweils eigenen Rechts. Damit kann dann der Rechtsvergleich auch für die Rechtspolitik und damit für die künftige Rechtsgestaltung durch den Gesetzgeber nützlich sein - freilich immer nur in recht begrenztem Umfang und stets unter dem Vorbehalt der Vereinbarkeit mit den jeweiligen nationalen Gegebenheiten, d. h. insbesondere den Traditionen (im Sozialrecht als zur Norm gewordenen Sozialpolitik auch den ,sozialpolitischen Pfaden") und der jeweiligen nationalen Rechtskultur, sowie den kulturellen, institutionellen, rechtlichen und politischen Rahmenbedingungen, den ökonomischen, personellen u. a. Ressourcen etc. Auf diese Weise können Anregungen, Beispiele und einschlägige Erfahrungen - nicht zuletzt ,good“ oder „,best practices“ - gewonnen und nutzbar gemacht werden für die Lösung rechtlicher Probleme. Der Rechtsvergleich kann überdies der Rechtsprechung benötigte Erkenntnisse dort vermitteln, wo ausländisches Recht berücksichtigt werden muss. ${ }^{3}$

In den Mitgliedstaaten der Europäischen Union stellt sich vor diesem Hintergrund heute die Aufgabe, vermehrt ausländisches Recht zu berücksichtigen und Rechtsver-

Der Beitrag beruht $u$. a. auch auf Teilergebnissen eines Forschungsprojekts, das der Verf. und andere Mitarbeiter des Max-Planck-Instituts für ausländisches und internationales Sozialrecht, München, zusammen mit dem Institut für Arbeitsmarkt- und Berufsforschung (IAB) der Bundesagentur für Arbeit, Nürnberg, und dem Institut für die Zukunft der Arbeit, Bonn (IZA), in den vergangenen beiden Jahren zur Aktivierungspolitik auf dem Arbeitsmarkt in ausgewählten Ländern - auch im Vereinigten Königreich: Finn, D./Schulte, B., ,Employment First': Activating the British Welfare State - durchgeführt haben, in: Eichhorst, W./Kaufmann, O./Konle-Seidl, R. (Hrsg.), Bringing the Jobless into Work? Experiences within Activation Schemes in Europe and the US, Berlin 2008, S. $297 \mathrm{ff}$.

2 So für den Sozialrechtsvergleich Zacher, H., Vorfragen zur Methode der Sozialrechtsvergleichung, in: ders. (Hrsg.), Methodische Probleme des Sozialrechtsvergleichs, Berlin 1977, S. 21 ff., 22; zu Begriff, Funktionen, Zielen und Methode der Rechtsvergleichung ausführlich Zweigert, K./Kötz, H., Einführung in die Rechtsvergleichung, 3. Aufl. Tübingen 1996, S. 5 f. m. w. N. Siehe auch den Beitrag von Becker, U., Rechtsdogmatik und Rechtsvergleich im Sozialrecht, in diesem Band, S. $11 \mathrm{ff}$.

3 Vgl. dazu etwa Eichenhofer, E., Sozialrechtsvergleichung und Sozialrechtspflege, Die Sozialgerichtsbarkeit (SGb) 1996, S. 257 ff.; Colneric, N., Die Rolle der Rechtsvergleichung in der Praxis des EuGH, in: Epiney, A. u. a. (Hrsg.), Die Herausforderung von Grenzen, Festschrift für Roland Bieber, Baden-Baden 2007, S. $316 \mathrm{ff}$. 
gleichung zu betreiben, sehr unmittelbar und sehr konkret im Zusammenhang mit der Auslegung und Anwendung Europäischen Rechts und der Konzipierung und Praktizierung Europäischer Politik, so etwa - um eine aktuelle Entwicklung anzusprechen - in der Sozialpolitik mit besonderer Bedeutung für die Fürsorge in ihrem aktuellen Gewand im Zusammenhang mit der sog. offenen Methode der Koordinierung (OMK) als seit „Lissabon 2000“ auch im Bereich des Sozialschutzes praktizierter politischer Strategie, die auf eine Annäherung der einschlägigen Politiken der Mitgliedstaaten der Europäischen Union durch wechselseitigen Informations- und Erfahrungsaustausch u. a. über „best practices“ im Bereich des Sozialschutzes angelegt ist und auf diese Weise soziale Ziele dort zu verwirklichen sucht, wo der Europäische Gesetzgeber mangels Kompetenz nicht tätig werden kann. ${ }^{4}$ Die „OMK Sozialschutz“ mit ihren Varianten OMK Soziale Inklusion, OMK Alterssicherung und OMK Gesundheitsversorgung und Langzeitpflege hat eine Vorläuferin in Gestalt der Europäischen Beschäftigungsstrategie (EBS), ${ }^{5}$ die ihren Platz im Beschäftigungstitel des EG-Vertrages gefunden hat und somit primärrechtlich verankert ist.

\section{Fürsorge/,, Social Assistance“ in der Europäischen Union}

Die Fürsorge bzw. Sozialhilfesysteme - „Social Assistance“-Systeme i. S. der von Karl-Jürgen Bieback in seinem Beitrag gewählten Begrifflichkeit - haben in der Europäischen Sozialpolitik einen spezifischen hohen Stellenwert im Rahmen der OMK Soziale Inklusion, die auf Eingliederung in die Gesellschaft (soziale Integration), auf Teilnahme und Teilhabe sowie eben auf Inklusion ${ }^{6}$,setzt“"

Im Weißbuch der Europäischen Kommission zu europäischem Regieren ${ }^{7}$ von 2001 wird die OMK wie folgt definiert:

4 Vgl. zur „offenen Methode der Koordinierung“ Schulte, B., Die Methode der offenen Koordinierung - Eine neue Strategie in der europäischen Sozialpolitik auch für den Bereich des sozialen Schutzes, ZSR 2003, S. 126 ff.; ders., Die „offene Methode der Koordinierung“ als politische Strategie in der Europäischen Sozialpolitik, Sozialer Fortschritt (SF) 2005, S. 105 ff.; ders., Die rechtlichen Rahmenbedingungen der offenen Koordinierungsmethode und ihr Einsatz in den unterschiedlichen Aktionsfeldern (Referat beim Centrum für Interkulturelle und Europäische Studien der Hochschule Fulda (Veröff. i. Vorb. 2009), jeweils m. w. N.; ferner die Beiträge von Danner, Devetzi, Eichhorst, Rhein, Karl und Streinz in: Deutscher Sozialrechtsverband (Hrsg.), Offene Methode der Koordinierung im Sozialrecht (Bundestagung 2004 in Aachen), Wiesbaden 2005.

5 Vgl. Art. 125-130 EG-Vertrag.

6 Vgl. zu diesem Begriff und seiner Bedeutung für die nationale und Europäische Sozialpolitik dazu Schulte, B., Soziale Inklusion im europäisch-vergleichenden Zusammenhang aus juristischer Sicht, Düsseldorf (Hans-Böckler-Stiftung) 2008, S. 7 ff. u. $120 \mathrm{ff}$.

7 Europäische Kommission, Weißbuch der Kommission über europäisches Regieren (KOM 2001/428 v. 25.7.2001), Brüssel 2001. 
„Die offene Koordinierungsmethode wird fallweise angewandt. Sie fördert die Zusammenarbeit, den Austausch bewährter Verfahren sowie die Vereinbarung gemeinsamer Ziele und Leitlinien von Mitgliedstaaten, die manchmal wie im Falle der Beschäftigung und der sozialen Ausgrenzung durch Aktionspläne von Mitgliedstaaten unterstützt werden. Diese Methode beruht auf einer regelmäßigen Überwachung der bei der Verwirklichung dieser Ziele erreichten Fortschritte und bietet den Mitgliedstaaten die Möglichkeit, ihre Anstrengungen zu vergleichen und aus den Erfahrungen der Anderen zu lernen.“

Die OMK ist ein Koordinierungsmechanismus für den nicht harmonisierten Bereich, in dem es keine Rechtsgrundlage für Legislativakte gibt; sie ist ein Mittel, einen Erfahrungsaustausch dort zu organisieren, wo die Europäische Union ansonsten keine oder nur beschränkte Kompetenzen hat.

Die Mitgliedstaaten sollen im Wege der OMK voneinander lernen. Die OMK ist auch die Grundlage für das Verfahren der politischen Abstimmung und Zusammenarbeit auf der Grundlage gemeinsamer Beschlüsse auf EU-Ebene. Sie ist hingegen keine materielle Grundlage dafür, die nationale Kompetenz über im Rat getroffene Vereinbarungen hinaus einzuschränken; es dürfen keine schleichenden Harmonisierungen stattfinden.

Die OMK ist eine Fortsetzung der Politik der Konvergenz von zu Beginn der 1990er Jahre: Unterscheidet man von einem quasi naturwüchsigen Prozess der Konvergenz im Sinne einer de-facto-Annäherung der Systeme des sozialen Schutzes der Mitgliedstaaten, welche auf der Existenz weitgehend übereinstimmender demografischer, ökonomischer, gesellschaftlicher, kultureller und internationaler Herausforderungen sowie gleichfalls weitgehend gemeinsamen politischen Überzeugungen, Werten, Zielen und Rahmenbedingungen der sozialen Sicherheit zurückzuführen ist, eine Politik der Konvergenz, so tritt in den letzten Jahren eine deutliche Tendenz zutage hin zu einer gezielten Annäherung der sozialpolitischen Zielsetzungen der Mitgliedstaaten und auch zu einer wachsenden Übereinstimmung darüber, was die zur Erreichung dieser Ziele eingesetzten Instrumente angeht, auch in Ansehung der Fürsorge/,Social Assistance“ und Aktivierungspolitik (,workfare“).

Die Europäische Kommission hat sich bereits in den 1990er Jahren für eine solche Politik der Konvergenz ausgesprochen, die darauf abzielt, die Mitgliedstaaten zu einer freiwilligen Abstimmung der Ziele und Politiken des Sozialschutzes zu veranlassen, da Sozialpolitik und Sozialrecht in der Europäischen Union grundsätzlich Angelegenheiten der Mitgliedstaaten geblieben sind und eine soziale Harmonisierung im Bereich des Sozialschutzes dementsprechend nicht stattgefunden hat, auf absehbare Zeit auch nicht stattfinden wird.

Einschlägige Rechtsinstrumente sind die Empfehlung 92/442/EWG über die Annäherung der Ziele und der Politiken im Bereich des sozialen Schutzes ${ }^{8}$ (,Konvergenzempfehlung") und die parallel dazu ergangene Empfehlung 92/441/EWG über gemeinsame Kriterien für ausreichende Zuwendungen und Leistungen im Rahmen der Systeme der

8 ABl. EG 1992 Nr. L 245 S. 46 v. 26.8.1992. 
sozialen Sicherung9 („Mindesteinkommensempfehlung“) aus dem Jahre 1992. Diese Empfehlungen sind auch heute noch Ausgangspunkt für Maßnahmen hinsichtlich der Bekämpfung von Armut und sozialer Ausgrenzung und betrifft damit unmittelbar auch die Fürsorge/,Social Assistance“-Systeme. ${ }^{10}$

Die Europäische Kommission definiert die soziale Inklusion als „Prozess, durch den gewährleistet wird, dass Personen, die von Armut und sozialer Ausgrenzung bedroht sind, die erforderlichen Chancen und Mittel erhalten, um am wirtschaftlichen, sozialen und kulturellen Geschehen teilzunehmen sowie in den Genuss des Lebensstandards und Wohlstands zu kommen, der in der Gesellschaft, in der sie leben, als normal gilt". Exklusion wird definiert als „Prozess, durch den bestimmte Personen an den Rand der Gesellschaft gedrängt und durch ihre Armut bzw. wegen unzureichender Grundfertigkeiten oder fehlender Angebote für lebenslanges Lernen oder aber infolge von Diskriminierung an der vollwertigen Teilhabe gehindert werden". 11

Für die Fürsorge/,Social Assistance/Minimum Income“-Systeme gilt, dass aufgrund spezifischer historischer Entwicklungen, unterschiedlicher ökonomischer, kultureller, rechtlicher und gesellschaftlicher Voraussetzungen sowie politischer Orientierungen die hier bestehenden Unterschiede zwischen den Mitgliedstaaten ${ }^{12}$ noch größer sind als im Bereich der sozialen Sicherheit (mit dem Kernbereich Sozialversicherung). Der Bereich der sozialen Sicherheit hat aufgrund zum einen länger andauernder und intensiverer staatlicher Regulierungen sowie der traditionellen, zu einer gewissen Vereinheitlichung

9 AB1. EG 1992 Nr. L 245 S. 49 v. 26.8.1992.

10 Vgl. Mitteilung der Europäischen Kommission „Modernisierung des Sozialschutzes im Interesse einer größeren sozialen Gerechtigkeit und eines stärkeren wirtschaftlichen Zusammenhalts, die aktive Einbeziehung der arbeitsmarktfernsten Menschen voranbringen, Brüssel, den 17.10.2007 (KOM (2007) 620 endg.).

11 Europäische Kommission, Gemeinsamer Bericht über die soziale Eingliederung, soziale Sicherheit und soziale Integration, Brüssel/Luxemburg 2004, S. 12.

12 Vgl. dazu Eardley, T. et al., Social Assistance in OECD-Countries. Vol. 1: National Reports; Vol. 2: Synthesis Report, London 1996; zu den unterschiedlichen Mindesteinkommens- bzw. Sozialhilferegelungen in den 12 Mitgliedstaaten Schulte, B., Mindesteinkommen in den Mitgliedstaaten der Europäischen Gemeinschaft - Nationaler Status quo und supranationale Initiativen, Sozialer Fortschritt (SF) 1991, S. 7-23; ders., Die wohlfahrtsstaatlichen Regimes in der EG, Zeitschrift für Sozialreform (ZSR) 1991, m. w. N.; ders., Armut und Armutsbekämpfung in der Europäischen Gemeinschaft Mindesteinkommenssicherung und Sozialhilfe in EG-Sozialrecht und EG-Sozialpolitik, ZfSH/SGB 1992, S. 393 ff.

$\mathrm{Zu}$ den europäischen Fürsorge/Social Assistance/minimum income-Systemen vgl. Guibentif, P./Bouget, D. (Hrsg.), Mindesteinkommen in der Europäischen Union - ein sozialpolitischer Vergleich, Lissabon 1997; Heikkilä, M./Kerkitalo, E. (Hrsg.), Social Assistance in Europa. A comparative study on minimum income in seven European countries, Helsinki 2001; Meyerhöfer, F., Soziale Mindeststandards in Europa, Frankfurt/M. 2000; Standing, G. (Hrsg.), Minimum Income schemes in Europe, Genf 2003; Moreira, A., The Activation Dilemma. Reconciling the fairness and affectiveness of minimum income schemes in Europe, Bristol 2008; jüngst Cantillon, B./Mechelen, $N$. van/Schulte, B., in: Minimum income policies in old and new member states, in: Alber, J./Fahey, T./Saraceno, Ch. (Hrsg.), Handbook of Quality of Life in the Enlarged European Union, London 2007, S. 218 ff. 
drängenden Strukturierung infolge der Anknüpfung entweder kategoriell an den Arbeitnehmerstatus (so im ausgehenden 19. Jahrhundert in der Ära der Entstehung der deutschen Arbeiterversicherung als der „Urform“ der Sozialversicherung kontinentaleuropäischen Typs) oder universell und damit in personeller Hinsicht umfassender an den Bürger-/Einwohnerstatus (so nach dem Zweiten Weltkrieg vor allem in Großbritannien und in den nordischen Ländern) und zum anderen wegen der Ausrichtung an den sowohl auf nationaler als auch auf internationaler Ebene (z. B. ausweislich des IAOÜbereinkommens Nr. 102 über die Mindestbedingungen der sozialen Sicherheit von 1952) anerkannten sozialen Risiken Krankheit, Mutterschaft, Invalidität, Alter, Tod (Hinterbliebenensicherung) und Familienlasten eine in den Grundzügen weitgehend einander angenäherten Konzepten und Prinzipien verpflichtete Ausrichtung gefunden.

Es kommt hinzu, dass die Systeme der sozialen Sicherheit der Mitgliedstaaten in Gestalt der Verordnungen (EWG) Nr. 14078/71 und Nr. 574/72 ein „Regime“ der sozialen Sicherheit der ein- und auswandernden Arbeitnehmer, Selbständigen und jüngst Studenten und ihrer Familienangehörigen eingeführt haben, das diesem Personenkreis die Zusammenrechnung anspruchsbegründender Beschäftigungs-, Versicherungs- und Wohnzeiten, Wahrung erworbener Rechte, Inländergleichbehandlung sowie Leistungsexport gewährleistet und das gleichsam eine gemeinsame Klammer für die im Übrigen nach wie vor eigenständigen nationalen Systeme gestaltet hat, die dazu beiträgt, dass diese Systeme in wachsendem Maße auf Annäherung angelegt sind (,,konvergieren“).

Da die Fürsorge/Sozialhilfe/,,Social Assistance“ gemäß Art. 4 Abs. 4 VO 1408/71 vom sachlichen Anwendungsbereich dieses Europäischen Koordinierungsrechts ausgenommen ist, entfällt für sie diese Klammerfunktion.

Auch die sonstigen Aktivitäten der Europäischen Gemeinschaft im Bereich des Sozialschutzes befassen sich in erster Linie mit dem Recht der sozialen Sicherheit unter Ausklammerung der Sozialhilfe, so dass von daher jedenfalls in der Vergangenheit kein Druck auf eine Annäherung der nationalen Grundsicherungs-/Sozialhilfesysteme im Europäischen Kontext ausgegangen ist.

Allerdings enthält die - als politische Erklärung zwar rechtlich unverbindliche, aber für die öffentliche Diskussion um den Stellenwert der Sozialpolitik in der Europäischen Gemeinschaft sehr wichtige - Gemeinschaftscharta der sozialen Grundrechte der Arbeitnehmer von 1989 auch ein Recht auf sozialen Schutz einschließlich einer sozialen Mindestsicherung. Danach - Ziffer 10 - hat jeder Arbeitnehmer „Anspruch auf einen angemessenen sozialen Schutz“, muss er ,unabhängig von seiner Stellung und von der Größe des Unternehmens, in dem er arbeitet, Leistungen der sozialen Sicherheit in ausreichender Höhe erhalten“ und müssen aber zugleich auch ,alle, die vom Arbeitsmarkt ausgeschlossen sind, weil sie keinen Zugang dazu fanden oder sich nicht wieder eingliedern konnten, und die nicht über Mittel für ihren Unterhalt verfügen“, ,ausreichende Leistungen empfangen und Zuwendungen beziehen können, die ihrer persönlichen Lage angemessen sind“. Des Weiteren muss - Ziffer 24 -, ,jeder Arbeitnehmer in der Europäischen Gemeinschaft, wenn er in den Ruhestand geht, über Mittel verfügen können, die 
ihm einen angemessenen Lebensstandard sichern“, und - Ziffer 25 - ,jeder, der das Rentenalter erreicht hat, aber keinen Rentenanspruch besitzt oder über keine sonstigen ausreichenden Unterhaltsmittel verfügt, ausreichende Zuwendungen, Sozialhilfeleistungen und Sachleistungen bei Krankheit erhalten können, die seinen spezifischen Bedürfnissen angemessen sind.“

Nach der vorerwähnten „Mindesteinkommensempfehlung“ sollen die Mitgliedstaaten sich unter Berücksichtigung ihrer jeweiligen innerstaatlichen Regelungen - in Deutschland seinerzeit der Sozialhilfe, in Frankreich des revenu minimum d'insertion (R.M.I.), in Großbritannien der supplementary benefits und des family credit - dazu verpflichten, für Personen, die ihren Lebensunterhalt nicht decken können, ausreichende Leistungen und Zuwendungen bereitzustellen, die ihrer persönlichen Lage entsprechen. Einvernehmen soll zwischen den Mitgliedstaaten insbesondere über folgende Punkte hergestellt werden:

- die Verankerung eines Rechtsanspruchs auf Leistungen zur Deckung des notwendigen Lebensunterhalts;

- die Einräumung eines derartigen Anspruchs an alle Personen, die sich rechtmäßig auf dem Staatsgebiet des jeweiligen Mitgliedstaats aufhalten;

- die Abhängigkeit dieses Anspruchs auf ein „,minimum vital“ von der Bedürftigkeit und einem entsprechenden Nachweis (Bedürftigkeitsprüfung/,,income-“ oder „means-test");

- die Gewährung der Leistungen unbegrenzt für die gesamte Dauer der Notlage;

- die Festsetzung der Leistung in Höhe des zur Deckung der elementaren Lebensbedürfnisse - des ,sozio-kulturellen Minimums“ - in dem jeweiligen Mitgliedstaat notwendigen Betrages;

- die Abhängigkeit des Leistungsanspruchs von der vorrangigen Verpflichtung zum Einsatz der Arbeitskraft im Hinblick auf eine zumutbare Erwerbstätigkeit als Form der Selbsthilfe;

- die Verknüpfung der Leistungen zur Sicherung des Lebensunterhalts mit solchen der beruflichen und sozialen Integration;

- die Bereitstellung einer angemessenen Unterkunft;

- die Berücksichtigung der familiären Situation des Hilfeberechtigten bei der Leistungsfestsetzung;

- die Anpassung der Leistungen an die Entwicklung der Preise und ggf. auch der Löhne, um eine Teilhabe am gesellschaftlichen Fortschritt zu ermöglichen;

- die Berücksichtigung besonderer Bedarfe insbesondere bei Krankheit, Behinderung, Alter, Pflegebedürftigkeit durch die Erhöhung der Regelleistungen oder die Gewährung zusätzlicher Leistungen;

- die Eröffnung des Zugangs zu medizinischen, rehabilitativen und sozialen Dienstleistungen sowie allgemein zu den in der jeweiligen Gesellschaft bestehenden Entfaltungsmöglichkeiten in Bezug auf Ausbildung, Beschäftigung, kulturelle Betätigung und Partizipation am gesellschaftlichen Leben; 
- die Bereitstellung ausreichender Informationen und Beratungsmöglichkeiten über diese Rechte, die Gewährleistung eines adäquaten Verfahrens sowie eines hinreichenden Rechtsschutzes zur Leistungsdurchsetzung.

Die in diesem Rechtsinstrument vorgesehene Gewährleistung eines sozialen Minimums für jedermann, die Förderung der beruflichen Eingliederung für diejenigen, die in der Lage sind, eine Erwerbstätigkeit auszuüben, sowie die soziale Eingliederung und die angemessene Absicherung zusätzlicher Bedarfe in entsprechenden Lebenslagen waren und sind zugleich Bestandteil der gleichzeitig vorgelegten und auch in die Form einer Empfehlung gekleideten Strategie zur Abstimmung der Ziele der Politiken des Sozialschutzes der Mitgliedstaaten (,Konvergenz-Empfehlung“). ${ }^{13}$

\section{Die Entwicklung des sozialen Schutzes in Deutschland und Großbritannien}

\section{19. Jahrhundert: Traditionelle nationale „Pfade“ der sozialpolitischen}

\section{Entwicklung}

In vergleichenden Darstellungen zur Sozialpolitik werden das deutsche und das britische System der sozialen Sicherung herkömmlicherweise als unterschiedliche Typen, ja Gegenpole dargestellt, etwa in Gestalt der geläufigen und einprägsamen (allerdings allzu vereinfachenden und vor allem der heutigen sozialpolitischen und sozialrechtlichen Systementwicklung nicht mehr entsprechenden) Unterscheidung zwischen „Bismarck-“ und „Beveridge-System“.

Blickt man zurück auf die Anfänge der modernen Sozialschutzpolitik im 19. Jahrhundert, so mag man die unterschiedlichen Bemührungen um eine soziale Absicherung der jeweiligen Bevölkerung in beiden Ländern als Gegensatz von „Arbeiterpolitik“ in Deutschland und „Armenpolitik“ in Großbritannien auf den Begriff bringen. ${ }^{14}$ Im ausgehenden 19. Jahrhundert standen sich dementsprechend institutionell „Arbeiterversicherung“ und „Poor Law“ gegenüber.

Das klassische britische Armenrecht („Poor Law“) von 1834 basierte auf drei Grundprämissen:

(i) dass jeder Arbeitsfähige auch Arbeit finden und deshalb für sich und seine Familie einen angemessenen Lebensstandard sicherstellen könne, so dass die Menschen des-

13 Vgl. zu dieser Konvergenz-Strategie Schulte, B., Abstimmung der Ziele der Politiken des Sozialschutzes in den Mitgliedstaaten der Europäischen Gemeinschaften - Ein weiterer Schritt auf dem Weg zur Sozialgemeinschaft? -, ZfSH/SGB 1991, S. 281 ff.

$14 \mathrm{Vgl}$. in diesem Sinne Leibfried, S./Tennstedt, F., Armenpolitik und Arbeiterpolitik, Zur Entwicklung und Krise der traditionellen Sozialpolitik der Verteilungsformen, in: dies. (Hrsg.), Politik der Armut und Die Spaltung des Sozialstaats, Frankfurt/Main 1985, S. 65 ff. 
halb in arbeitsfähige, die nur Hilfe erhielten, wenn sie die Härten des „workhouse“ akzeptierten, sowie arbeitsunfähige Personen - Alte, Behinderte und Kranke - eingeteilt wurden, die auch Hilfe außerhalb des Arbeitshauses erhalten konnten;

(ii) dass die Hilfe im „,workhouse“ abschreckend und deshalb so wenig attraktiv sein sollte, dass jemand, der sie in Anspruch nahm, auch wirklich über keine andere Einkommensquellen verfügte (wobei dann ggf. das „Arbeitshaus“ durch einen sog. „labour test" ersetzt wurde: es gab Hilfe „draußen“ als Gegenleistung für die Ableistung von Arbeit); sowie

(iii) auf dem Grundsatz der „less eligibility“, wonach, um den Anreiz zur Arbeit aufrechtzuerhalten, die Unterstützungsleistungen den „Lohn des am schlechtesten bezahlten Arbeiters" nicht übersteigen durften.

Die deutsche Sozialversicherungsgesetzgebung der 1880er Jahre hatte in Großbritannien zunächst keine Entsprechung; es dauerte noch dreißig Jahre, bevor hier - im Jahre 1911 - eine Sozialversicherung eingeführt wurde. Dies geschah durchaus im Wissen um die deutsche Lösung: William Beveridge hatte im Jahre 1905 Deutschland besucht, um das dort errichtete System der Arbeitsvermittlung zu studieren. Im Jahre 1908 war Lloyd George nach Deutschland gereist, um sich über die deutsche Arbeiterversicherung zu informieren. Der Besuch von William Beveridge in Deutschland im Jahre 1905 hat in seiner grundlegenden Schrift „Unemployment: A Problem of Industry“ von 1909 seinen Widerhall gefunden. Der sehr früh und sehr vielseitig tätige Winston Churchill, seinerzeit Präsident des Board of Trade, hat sich 1908 für einen „Schuss ,Bismarckianism” “ in Großbritannien ausgesprochen.

Nach der Jahrhundertwende erkannte man in Großbritannien mehr und mehr, dass das „Poor Law“ keine geeignete Lösung für jene bereithielt, die auf Dauer arbeitslos waren, beschränkte sich die Hilfe des Staates doch darauf, diese Personen ,über Wasser zu halten", bis sie selbst wieder Arbeit fanden. ${ }^{15}$

Im Jahre 1911 sind zwei unabhängig verwaltete staatliche Versicherungssysteme eingeführt worden: Das erste war eine Krankenversicherung als Pflichtversicherung für (mit einigen Ausnahmen) abhängig beschäftigte manuelle Arbeiter zwischen 16 und 70 sowie für sonstige abhängig Beschäftigte, die weniger als ein bestimmtes Minimum verdienten, sah medizinische Behandlung und pauschale Geldleistungen bei Krankheit, Mutterschaft und Invalidität vor; das zweite war eine Arbeitslosenversicherung, die gleichfalls pauschale Geldleistungen für eine Zeit der Arbeitslosigkeit von maximal 15 Wochen pro Jahr für Arbeiter aus bestimmten Sektoren der Industrie vorsah.

Dementsprechend unterschied sich die britische Volksversicherung, die durch den National Insurance Act 1911 fast drei Jahrzehnte später als die deutsche Arbeiterversicherung eingeführt worden ist, grundlegend von ihrem deutschen Gegenstück (das in demselben Jahr in Gestalt der Reichsversicherungsordnung, die dann ihrerseits Ende des 20. Jahrhunderts durch das Sozialgesetzbuch abgelöst wurde, kodifiziert worden ist).

15 Beveridge, $W$. ., Unemployment: A Problem of Industry, London 1909. 
Die Health insurance sah Krankenpflegeleistungen sowie einheitliche, d. h. nicht wie in Deutschland einkommens- bzw. lohnbezogene Geldleistungen bei Krankheit, Mutterschaft und - wiederum anders als in Deutschland, wo entsprechende Regelungen ihren Platz in der Rentenversicherung gefunden hatten - auch bei Invalidität vor. Die Unemployment insurance hatte seinerzeit in Deutschland keine Entsprechung.

Die Arbeitslosigkeit war mithin zusammen mit der Krankheit das erste soziale Risiko, das durch die Sozialversicherung in Großbritannien abgedeckt wurde. Großbritannien war damit insofern ein Pionier in Europa, als anderswo - in Deutschland im Jahre 1922 - Arbeitslosenversicherungssysteme erst später eingeführt wurden.

Ein Antragsteller auf Leistungen bei Arbeitslosigkeit hatte nicht von vornherein einen Anspruch auf Leistungen vom ersten Tag der Arbeitslosigkeit an, sondern er musste erst eine Wartezeit von drei „waiting days“ zurücklegen. Der Gesetzgeber ging davon aus, dass auch ein Arbeitsloser normalerweise ausreichende Mittel zur Verfügung habe, um eine kurze „Durststrecke“ zu überbrücken, und dass die Verwaltungskosten bei der Gewährung von kurzfristigen Leistungen unverhältnismäßig hoch wären.

Der Antragsteller musste für eine mögliche Vollzeitbeschäftigung zur Verfügung stehen und wurde vorübergehend vom Leistungsbezug ausgeschlossen, wenn er aufgrund eigenen Verschuldens seine Stelle verloren hatte oder keinen anderen Arbeitsplatz finden konnte.

Da das Auftreten von Arbeitslosigkeit schwer vorherzusagen ist und die britische Arbeitslosenversicherung im Jahre 1911 weltweit ein Novum war, fühlten sich seine Urheber bewogen, hinsichtlich der Frage, welcher Teil der Arbeitslosenpopulation erfasst werden sollte, zurückhaltend zu sein: Berücksichtigt wurden Bauwesen, Schiffsbau, Ingenieurwesen, Fahrzeugbau, Eisengießereien und bestimmte Arbeiten von Sägewerken - Branchen, denen gemeinsam war, dass sie eher zyklischer und saisonbedingter Arbeitslosigkeit ausgesetzt waren als chronischer Arbeitslosigkeit und Unterbeschäftigung.

Ein ,großer Sprung nach vorn“ fand dann im Jahre 1920 statt, als die Arbeitslosenversicherung auf alle Personen erstreckt wurde, die einen Arbeits- oder Lehrlingsvertrag hatten (freilich immer noch mit gewissen Ausnahmen: Landwirtschaftliche Arbeiter, im Haushalt Beschäftigte, Mitglieder der Streitkräfte und Beamte, Lehrer und andere nicht manuell tätige Arbeitnehmer mit einem Verdienst von über £ 250 pro Jahr ${ }^{16}$ ).

Zur Altersversorgung war im Unterschied zur deutschen Rentenversicherung durch den Old Age Pensions Act 1908 drei Jahre zuvor ein aus Steuermitteln finanziertes, beitragsfreies und nicht auf dem Versicherungsprinzip beruhendes Altersrentensystem eingeführt worden.

Das britische Social Security-System - auch in der Gestalt, die es rd. drei Jahrzehnte später durch die Umsetzung des sog. Beveridgeplans ${ }^{17}$ von 1942 nach dem Zweiten

16 National Insurance Act (NIA) 1911, s (= section) 6.

17 Vgl. Beveridge, W., Social Insurance and Allied Social Services, London 1942 (deutschsprachige Ausgabe: Der Beveridgeplan, Bern 1943). 
Weltkrieg in den Jahren zwischen 1945 und 1948 gefunden hat - war von Anbeginn an und ist bis heute bedarfsorientiert und grundsichernd (was dem britischen Welfare State das Etikett des „liberalen“ Wohlfahrtsstaats eingetragen hat der - vielleicht allzu vereinfachend - dem sog. korporatistischen Wohlfahrtsstaat kontinentaleuropäischer und damit auch deutscher Provenienz sowie dem sozialdemokratischen Wohlfahrtsstaatstyp der nordischen Staaten gegenüber gestellt worden ist). ${ }^{18}$

Insofern entstand das britische System der sozialen Sicherheit als Alternative zum klassischen Armenrecht, dem „Poor Law“ des 19. Jahrhunderts. Denn im Unterschied zum privaten Versicherungsmarkt ${ }^{19}$ beruhte die National Insurance von Anfang an auf einem System einheitlicher Beiträge und Leistungen und folgte nicht dem versicherungstechnischen Äquivalenzprinzip mit seiner Entsprechung von Beitrag und Leistung wie die beitragsfinanzierten Einkommensersatzleistungen in Deutschland.

\section{20. Jahrhundert: Entstehung und Entwicklung von Welfarestate und}

\section{Sozialstaat}

Die staatlich verantworteten sozialen Sicherungssysteme sind in Großbritannien im Unterschied zu Deutschland von Anbeginn an sehr zentralistisch ausgestaltet und verwaltet worden und umfassen seit Umsetzung des Beveridgeplans im Grundsatz die gesamte Erwerbsbevölkerung - der staatliche Gesundheitsdienst die gesamte Wohnbevölkerung -, ergänzt „,nach oben“ durch für bestimmte Gruppen der Bevölkerung geschaffene betriebliche (occupational pension schemes) und private Zusatzsicherungssysteme (private provision) als zweite und dritte Säule der Alterssicherung sowie „nach unten“ durch bedarfsorientierte Leistungen, insbesondere die staatliche Fürsorge - „National Assistance“, „Social Assistance“, „Supplementary Benefits“ (ergänzt durch „Family Credit“ und zuletzt ,contribution-based' und ,income-based' Income Support sowie „tax credits“" (Steuergutschriften) als jeweils unterschiedliche systemische Ausprägungen).

Die Unterscheidung von social, fiscal und occupational welfare als Typen sozialpolitischer Intervention kennzeichnet die soziale Sicherung i. w. S. in Großbritannien als das Ergebnis eines ,policy mix“, d. h. des Zusammenwirkens vielfältiger - spezifischer wie unspezifischer, monetärer wie nicht-monetärer, öffentlicher wie privater - Maßnahmen. Das britische social welfare system setzt sich aus sozialen Diensten, staatlichem Gesundheitsdienst und Einkommenssicherung zusammen. Während die personal

18 Vgl. krit. zu dieser Typologie, die auf Gösta Esping-Andersen (The Three Worlds of Welfare Capitalism, Oxford 1995) zurückgeht, Schulte, B., Sozialstaat und Europäische Union, in: Clever, P./Schulte, B. (Hrsg.), Bürger Europas, Bonn 1995, S. 62 ff. m. w. N.; auch ders., The Welfare State and European Integration, European Journal of Social Security (EJSS) 1995, S. 7 ff.

19 Vgl. so etwa Atkinson, A., Social Insurance, in: Geneva Papers on Risk and Social Insurance Theory 1991, S. 2; Alcock, P., Vor- und Nachteile der Beitragsgrundlage bei der Steuerung von Leistungen: Eine Sozialanalyse des Versicherungssystems im Vereinigten Königreich, Internationale Revue für Soziale Sicherheit (I.R.S.S.) 49 (1996), S. 33 ff., 34. 
social services im Wesentlichen in den Aufgabenbereich der kommunalen Gebietskörperschaften (local authorities) fallen und auch die Freie Wohlfahrtspflege (voluntary organizations) hier ein Betätigungsfeld hat, stehen National Health Service und Social Security in der Verantwortung des Gesundheitsministeriums und des Ministeriums für Soziale Sicherheit (Department of Health and Social Security), an dessen Spitze ein Minister steht. Nachgeordnet sind regionale und lokale Ämter, jeweils getrennt für die Bereiche Gesundheit und Soziale Sicherheit. Erziehungswesen (Education) und Wohnungswesen (Housing) zählen nicht zum Sozialleistungssystem, sind aber zu erwähnen, weil insbesondere der letztgenannte Bereich traditionellerweise durch Wohnbeihilfen in erheblichem Maße zur Verbesserung der Einkommenslage der Bürger beiträgt.

Die Social Security gewährt sozialen Schutz durch monetäre Leistungen bei Krankheit, Mutterschaft, Invalidität, Arbeitsunfall und Berufskrankheit, Arbeitslosigkeit, Alter und Tod (Hinterbliebenensicherung) sowie Familienleistungen.

Nach dem Zweiten Weltkrieg wurden in Ausführung des Beveridgeplans die bestehenden staatlichen Sozialversicherungssysteme zu einem einzigen System zusammengefasst, das einem Ministry of National Insurance unterstand. Wichtigste Neuerungen waren die Erstreckung der Pflichtversicherung auf alle Mitglieder der Bevölkerung im arbeitsfähigen Alter - eingeteilt in abhängig Beschäftigte, Selbständige, NichtErwerbstätige und verheiratete Frauen -, ein nationaler Gesundheitsdienst sowie ein staatliches Versicherungssystem für Arbeitsunfälle und Berufskrankheiten.

Das auf Reziprozität angelegte ,welfare“-Modell mit seiner Betonung auf dem Recht auf Leistungen, das sich die Bürger durch ihre Beiträge erwarben, war ein zentraler Punkt der Beveridge-Philosophie: „Leistungen gegen Beiträge anstatt freie Beihilfe vom Staat ist das, was die Menschen in Großbritannien wünschen." Die Gewährung verdienstbezogener Leistungen, die bei den kontinentaleuropäischen Sozialversicherungssystemen üblich war, war der britischen Tradition und auch den Reformvorstellungen von Beveridge fremd. Der Einzelne sollte vielmehr selber Vorsorge jenseits des Existenzminimums für sich selbst treffen; Instrument zur Umverteilung etwa durch die Koppelung verdienstbezogener Beiträge mit Pauschalleistungen sollte die Volksversicherung nicht sein.

Der überkommene Grundsatz der einheitlichen (,flat-rate") Beiträge ließ sich mit der liberalen Philosophie in Einklang bringen: Beiträge, die entsprechend der wirtschaftlichen Leistungsfähigkeit variieren, würden danach zu einer wirtschaftlichen Umverteilung führen; stattdessen wurden ,arm“ und „reich“ gleich behandelt. Diese Strategie verlangte jedoch, dass die Beiträge in einer Höhe festgesetzt wurden, die auch gering entlohnte Versicherter noch aufbringen konnten, was - sollten nicht die Beiträge der Arbeitgeber und die staatlichen Zuschüsse erhöht werden - zwangsläufig die Höhe der Geldleistungen und zumal der Altersrenten nach oben begrenzten. Dies war dann auch der Hauptgrund für den Fehlschlag der Volksversicherung bereits in den 1950er Jahren in Gestalt der Herausbildung von „two nations in old age“ - jeder dritte Altersrentner 
war auf ergänzende Fürsorgeleistungen angewiesen - sowie in den 1960er Jahren dann für die Ersetzung der „flat-rate“ durch einkommensbezogene Beiträge.

Die Sozialversicherungssysteme der Vorkriegszeit waren zu unterschiedlichen Zeiten und mit unterschiedlichen Zielen errichtet worden. Im Interesse der Verwaltungseffizienz sollte es künftig einen einzigen Fonds geben, an den die Versicherten einen einheitlichen (im Allgemeinen wöchentlich zu entrichtenden) Gesamtbeitrag leisteten, sowie eine gemeinsame Organisation zur Bereitstellung von Leistungen. Die gesamte Bevölkerung sollte künftig gegen alle großen sozialen Risiken geschützt werden. Ihre Klassifizierung in Beitragsgruppen - abhängig Beschäftigte, Selbständige, Nichterwerbstätige u. a. - war notwendig, um Unterschieden im Hinblick auf die Bedürftigkeit sowie dem unterschiedlichen sozioökonomischen Status einzelner Gruppen der Bevölkerung angemessen Rechnung zu tragen.

Die britische Volksversicherung in der Gestalt, die sie durch den National Insurance Act 1946 erhalten hatte, zeichnete sich dementsprechend dadurch aus, dass gemäß dem Grundsatz der Vollständigkeit (,comprehensiveness") des Beveridge-Plans grundsätzlich die gesamte Bevölkerung in ein und dasselbe Versicherungssystem entweder obligatorisch einbezogen wird oder doch - in bestimmten eng umgrenzten Fällen - das Recht zum freiwilligen Beitritt hat (sodass man insbesondere auch angesichts des hohen Deckungsgrades der beitragsbezogenen Leistungssysteme, in Sonderheit auch, was nicht erwerbstätige Personen angeht, die britische Sozialversicherung den universellen Systemen sozialer Sicherung zuordnen kann).

Einkommensbezogene Zulagen wurden von $1966^{20}$ an zusätzlich zu Arbeitslosengeld und Krankengeld gewährt.

Der Social Security Act 1975 ersetzte das System der Einheitsbeiträge durch ein einkommensbezogenes Beitragssystem.

Seitdem wird auch zwischen beitragsbezogenen (contributory benefits) und beitragsfreien Leistungen (non-contributory benefits) unterschieden: Erstere bezeichnen die Leistungen der früheren Volksversicherung (National Insurance) und lassen sich als Vorsorgesysteme nach deutscher Systematik begreifen, die letztgenannten bezeichnen eine Reihe steuerfinanzierter Leistungen, die man teils als soziale Entschädigungssysteme, teils als soziale Hilfs- und Förderungssysteme qualifizieren kann. In den Genuss beitragsfreier Leistungen kommen insbesondere Personen, die keinen Anspruch auf beitragsbezogene Leistungen haben, weil sie außerstande sind, Beiträge zu entrichten.

Aus Steuermitteln finanziert werden auch die konkret bedarfsabhängigen Sozialleistungen, i. e. die von einer Bedarfsprüfung abhängigen Einkommensunterstützungen (income support), die fürsorge- und sozialhilfeähnlichen Familiengutschriften (family credit) und die - gleichfalls fürsorge- bzw. sozialhilfeähnliche - Wohnbeihilfe (housing benefit).

20 National Insurance Act (NIA) 1966, s. 2. 
Abstrakt bedarfsorientiert sind die steuerfinanzierten Leistungssysteme, die im $\mathrm{Zu}-$ sammenhang mit der Belastung durch den Unterhalt von Kindern ohne besondere Prüfung einen Bedarf als vorliegend unterstellen und ihn (teilweise) decken (child benefit).

Die Einführung arbeitseinkommensbezogener Leistungszuschläge unter Labour in den 1970ern war nicht nur gleichsam die Hochzeit des britischen Wohlfahrtsstaats, sondern hat das britische System der sozialen Sicherheit seinerzeit den charakterisierten kontinentaleuropäischen Systemen - und damit auch deutschen Sozialversicherungsmodell - ein Stück weit in Richtung auf das sozialpolitische Ziel der Lebensstandardsicherung angenähert (ohne dass es freilich zu einer „Hochzeit“ im Sinne einer Verbindung und Vereinigung der Systeme gekommen wäre). Das britische System der sozialen Sicherheit hat dann in den 1980er Jahren die tiefgreifendste Veränderung seit der im Gefolge des Beveridge-Plans vorgenommenen Umgestaltung nach Beendigung des Zweiten Weltkrieges erfahren.

Die 1979 ins Amt gekommene konservative Regierung unter Margret Thatcher setzte (nach dem „Maggie“ zugeschriebenen Bonmot „Von Marx and Engels to Marks \& Spencer") eine Wende in der Wirtschafts-, Beschäftigungs- und Sozialpolitik durch, die eine Abkehr von der Politik jeder anderen britischen Regierung einschließlich der früheren konservativen Regierungen seit Kriegsende bedeutete. Vor allem darauf gerichtet, die öffentlichen Ausgaben zu reduzieren und das Bewusstsein individueller Verantwortung zu revitalisieren, wurde eine Sozialgesetzgebung auf den Weg gebracht, die Ansprüche und Aussichten von Leistungsempfängern des sozialen Sicherheitssystems sehr einschränkte. Die davon am meisten Betroffenen waren die Empfänger kurzfristiger Leistungen bei Arbeitslosigkeit und Krankheit. Die Regierung suchte diese Politik damit zu rechtfertigen, dass die meisten Arbeitnehmer in der Vergangenheit bereits Versicherungsschutz gegen diese Risiken durch betriebliche Vorsorgeeinrichtungen erworben hätten, allzu großzügige Leistungen zu Missbrauch geführt hätten und schließlich größere Arbeitsanreize geschaffen werden müssten, was dann nicht möglich sei, wenn man außerhalb des Arbeitsprozesses materiell besser gestellt sei als wenn man erwerbstätig sei. Die wichtigsten Maßnahmen waren Reduzierung und Streichung lohnbezogener Zuschläge zu kurzzeitigen Leistungen und eine Neuregelung - sprich: Einschränkung des Leistungsbezugs. Die Anpassung von Geldleistungen wurde dahin modifiziert, dass diese Leistungen künftig weniger erhöht wurden als der Anstieg des Lebenshaltungskostenindexes.

„New Labour" unter Tony Blair hat die Reformen der Konservativen fortgeschrieben, wenn auch punktuell „sozial abgefedert“, etwa durch Einführung eines nationalen gesetzlichen Mindestlohns, die Einführung von Steuergutschriften „to make work pay“ und von Maßnahmen zur Bekämpfung namentlich der Kinderarmut. Instrumente der Beschäftigungsförderung sind verstärkt und die Anforderungen an die Zumutbarkeit von Beschäftigungsmöglichkeiten und an die Arbeitsuche sind verschärft worden. 


\section{IV. „Fürsorge“/,, Social Assistance“ als Teil der sozialen Sicherheit}

Im Jahre 2003 war in Deutschland das Gesetz über eine bedarfsorientierte Grundsicherung im Alter und bei Erwerbsminderung $(G S i G)$ in Kraft getreten und seine Regelungen waren neben die Sozialhilfe nach dem Bundessozialhilfegesetz getreten. Das Grundsicherungsgesetz sollte in erster Linie die sog. ,,verschämte Altersarmut“ bekämpfen und älteren und erwerbsunfähigen Personen, die nicht mehr zur Ausübung einer Erwerbstätigkeit verpflichtet waren, den „Gang zum Sozialamt“ ersparen. Im Zuge der Neugestaltung des Sozialhilferechts ist dieses Gesetz dann aufgehoben und seine Regelungen sind - in modifizierter Form - als Viertes Kapitel ( $\S \S 41-46)$ in das Sozialgesetzbuch (SGB) - Zwölftes Buch (XII) integriert worden. ${ }^{21}$

Bei Inkrafttreten des Bundessozialhilfegesetzes im Jahre 1962 schien angesichts der seinerzeit herrschenden Vollbeschäftigung Armut nur noch residual und im Wesentlichen Altersarmut zu sein. Dies hatte sich bis Ende der 90er Jahre entscheidend geändert: Damals bis heute waren nur rd. 1,3 v. H. der über 65-Jährigen auf ergänzende Hilfe zum Lebensunterhalt angewiesen gegenüber 3,5 v. H. der Gesamtbevölkerung. ${ }^{2} 2$

Die aktuelle Entwicklung der Alterssicherung dürfte wohl dazu führen, dass Altersarmut erneut zu einem Thema wird, nähert sich doch das von der Gesetzlichen Rentenversicherung (GRV) garantierte Sicherungsniveau ,von oben“ dem Niveau der Hilfe zum Lebensunterhalt der Sozialhilfe an. Diese durch die faktische Aufgabe des Ziels der Lebensstandardsicherung durch die GRV entstandene und künftig entstehende Sicherungslücke nicht in jedem Fall durch Maßnahmen auf der zweiten oder dritten - betrieblichen oder privaten - Ebene, d. h. durch Betriebsrenten und/oder Formen der privaten Vorsorge ausgeglichen werden können. Hier steht eine Renaissance der Fürsorge in modernem Gewand zu erwarten.

Diese bedenkliche armutspolitische und fürsorgerechtliche Entwicklung wäre noch offenkundiger, wenn die Regelsätze der Sozialhilfe und das Niveau der Grundsicherung, wie es das Bedarfsdeckungsprinzip als zentrales Strukturprinzip der Fürsorge/Sozialhilfe/Social Assistance an sich verlangt hätte, diesem tragenden Strukturprinzip fortgeschrieben und der Höhe nach angepasst - d. h. angehoben - worden wären, weil sich dann die „Schere“ zwischen Rentenniveau und Sozialhilfeniveau noch sehr viel weiter geschlossen hätte.

Stattdessen ist die Fortschreibung der Regelsätze in den letzten Jahren unter Bezugnahme auf die Entwicklung der Altersrenten mit der Konsequenz erfolgt, dass wie die

21 BGBl. 2004 I, S. 3022 ff.; zum Werdegang des Gesetzes vgl. Hummel, M., Das Gesetz über eine bedarfsorientierte Grundsicherung im Alter und bei Erwerbsminderung (GSiG) - ein tauglicher Ansatz zum Abbau „,verschämter Armut“ und zum Aufbau einer bedarfsgerechten Grundsicherung, ZFSH/SGB 2001, S. 713 ff.; Dünn, S./Rüpp, K., Die neue Regelung der Bedarfsorientierten Grundsicherung im Alter und bei Erwerbsminderung zum 1. Januar 2005, DRV 2004, S. 1614 ff.

22 Vgl. Ruland, F., Sozialhilfe als Grundsicherung?!, NJW 2000, S. 325 ff.; ders., Schwerpunkte der Rentenreformen in Deutschland, NJW 2004, S. 1273 ff. 
Rentner auch die Sozialhilfeempfänger in den letzten Jahren wirtschaftliche Einbußen erlitten haben - letztere allerdings insofern zwangsläufig schmerzlichere Einbußen, als die ihnen zustehenden Leistungen zur Sicherung des sozialen Existenzminimums dienen und insofern im wahrsten Sinne des Wortes „existenzieller“ sind als die Renten, die zum einen - jedenfalls früher - der Lebensstandardsicherung und heute immer noch der „gehobeneren“ sozialen Sicherung dienen sollen, und die zum anderen häufig nicht die einzigen Einkünfte der Rentenbezieher sind.

Diese Entwicklung stellt nicht nur die Gesetzliche Rentenversicherung (GRV) vor Legitimationsprobleme (,Sollte nicht die beitragsfinanzierte Vorsorge durch die GRV ein deutliches Mehr an Leistungen bringen gegenüber lediglich das Existenzminimum garantierenden steuerfinanzierten Sozialhilfesystem, damit sich Erwerbsarbeit sowie Eigenvorsorge in der Sozialversicherung auch aus Sicht der Betroffenen lohnen?"), sondern nötigt auch dazu, die Frage zu beantworten, inwiefern die heutigen Mindestsicherungsleistungen zum einen auf nationaler Ebene den verfassungs- und sozialrechtlichen und zum anderen auf Europäischer Ebene den EG-armutspolitischen (z. B. „60 \% des jeweiligen nationalen Durchschnittseinkommens") Vorgaben für die Gewährleistung des Lebensnotwendigen noch entsprechen.

Auch ist nach einem rechtsstaatlichen Verfahren zur regelrechten Anpassung des Existenzminimums nach Bedarfsgesichtspunkten und einer demokratisch legitimierten, ggf. parlamentarischen Festsetzung dieses nationalen sozialen Minimums zu fragen.

Die Bundesregierung hat in ihrem Bericht über die Höhe des Existenzminimums $2005^{23}$ darauf hingewiesen, dass die im SGB XII geregelte Hilfe zum Lebensunterhalt auch für das Steuerrecht maßgebliche Existenzminimum darstellt und insofern Referenzsystem auch für die Leistungen nach dem SGB II ist.

Das Bundesverfassungsgericht hat vor Jahren bereits zur Festlegung des steuerrechtlichen Existenzminimums im Einkommenssteuerrecht insbesondere auch im Zusammenhang mit Familienlastenausgleich auf die Hilfe zum Lebensunterhalt - seinerzeit nach Maßgabe des Bundessozialhilfegesetzes - verwiesen. Diese Bezugnahme auf die Sozialhilfe erscheint heute nicht unproblematisch wegen der geringen und wenig repräsentativen Zahl der Noch-Sozialhilfeempfänger und der bereits angesprochenen (verfassungsrechtlich bedenklichen) Nichtbeachtung des Bedarfsdeckungsprinzips bei der Regelsatzanpassung in den letzten Jahren. ${ }^{24}$

Die soziale Grundsicherung wird heute in Deutschland im Übrigen von vier statt wie früher zwei unterschiedlichen Regelungskomplexen gewährleistet (und ist damit de facto wie ehedem die klassische Fürsorge vor Inkrafttreten des Bundessoziahilfegesetzes „Gruppenfürsorge“):

23 Bundesregierung, Bericht über die Höhe des Existenzminimums von Erwachsenen und Kindern für das Jahr 2005 (Fünfter Existenzminimumbericht), BT-Dr. 15/2462 v. 5. 2. 2004, S. 2.

24 Vgl. zum Sozialhilferecht aktuell, instruktiv und umfassend Trenk-Hinterberger, P., Sozialhilferecht, in: Maydell, B. von/Ruland, F./Becker, U. (Hrsg.), Sozialrechtshandbuch (SRH), 4. Aufl. Baden-Baden 2008, S. 102 ff. 
(i) der Grundsicherung für Arbeitsuchende nach dem SGB II;

(ii) der Grundsicherung im Alter oder bei Erwerbsminderung nach dem SGB XII - 4 . Kapitel - gleichsam als neuer ,gehobener“ Fürsorge (nach klassischem Verständnis);

(iii) der überkommenen Hilfe zum Lebensunterhalt nach dem SGB XII - 3. Kapitel für einen heute gegenüber früher sehr eingeschränkten (rd. $10 \mathrm{v}$. H. Personenkreis); und

(iv) „ganz unten“ dem gegenüber der Sozialhilfe (mit fragwürdiger und auch verfassungsrechtlich bedenklicher Begründung) leistungsmäßig reduzierten „minimum minimorum" des Asylbewerberleistungsgesetz für dessen spezifische Klientel.

Die sozialpolitische Berechtigung und die sozialrechtliche „Stimmigkeit“ dieser Ausgestaltung der sozialen Grundsicherung ist zu hinterfragen: Ist beispielsweise Alter allein ein Grund, einen älteren Hilfebedürftigen gegenüber einem erwerbsfähigen Alleinerziehenden mit familiärer Verantwortung - z. B. einer ggf. unfreiwillig, nämlich etwa wegen fehlender Kinderbetreuungsmöglichkeit nicht erwerbstätigen alleinerziehenden Mutter mit Kindern, die auf Leistungen der Sozialhilfe i. e. S. angewiesen ist besser zu stellen?

Es gibt eine ganze Reihe von Inkonsistenzen, die es nahe legen, den Bereich der Mindestsicherung auch durch Vergleich mit den diesbezüglich auf eine längere Tradition zurückblickenden Nachbarstaaten, die i. d. R. Mindestsicherungskomponenten auch in der ,gehobenen“ sozialen Sicherung, d. h. insbesondere in den Sozialversicherungssystemen, kennen, zu überdenken.

$\mathrm{Ob}$ die Leistungen zur Sicherung des Lebensunterhalts im Rahmen der Grundsicherung für Arbeitsuchende vom Gesetzgeber adäquat festgesetzt worden ist, ist allerdings bundesverfassungsgerichtlich noch nicht geklärt worden. Eine diesbezügliche Verfassungsbeschwerde, die sich gegen die Festsetzung des Arbeitslosengeldes II nach $\S 20$ SGB II richtete, hat das Bundesverfassungsgericht im November 2007 vor allem wegen unzureichender Begründung gemäß $§ 23$ Abs. 1 S. 2, 92 BVerfGG als unzulässig nicht zur Entscheidung angenommen, ohne sich mit dieser in der Fachöffentlichkeit gegenwärtig vieldiskutierten Problematik auseinandersetzen. ${ }^{25}$

In der bayerischen Landeshauptstadt München ist seit 1. Mai 2008 der Regelsatz der Sozialhilfe von 347 auf 371 Euro angehoben worden unter Berufung auf ein Gutachten, das diesen höheren Satz als zur Bedarfsdeckung notwendig erachtet hat angesichts der Lebenshaltungskosten in einer teueren Metropole.

Auch die Festsetzung des Existenzminimums auf rechtstaatlich angemessene Weise - wegen der Wesentlichkeit dieser Entscheidung für die Betroffenen ggf. in einem parlamentarischen Verfahren - stellt nach wie vor eine Herausforderung für den deutschen

25 Beschluss der 3. Kammer des 1. Senats v. 7.11.2007 - 1 BVR 1840/07 - m. krit. Anm. v. K.-J. Bieback, NZS 2008, S. 409 ff.

Das LSG Hessen hält die Regelsatzregelung des SGB II wegen Verstoßes gegen das Menschenwürdeprinzip, den Gleichheitssatz und das Sozialstaatsprinzip, für verfassungswidrig und hat im Oktober 2008 deshalb das Bundesverfassungsgericht angerufen. Auch das BSG hat jüngst die Vereinbarkeit der Hartz IV-Regelung mit den Vorgaben der Verfassung verneint. Das BVerfG ist aufgerufen, hierüber letztverbindlich zu entscheiden. 
Gesetzgeber dar, zumal die Sozialhilfe auch Referenzgröße für „Minima“ in anderen Bereichen - z. B. im Steuerrecht - ist; auch wenn die jüngsten Reformen die Einheitlichkeit dieses sozialrechtlichen „Basic Floor“ durch ein Nebeneinander von Grundsicherung zum einen für Arbeitsuchende und für alte und erwerbsunfähige Personen und zum anderen „residualer“ Sozialhilfe i. e. S. für nicht erwerbsfähige - und zu einer Erwerbstätigkeit verpflichtete Personen sowie - sie voraussetzungs- wie leistungsmäßig noch unterbietender - Leistungen nach dem Asylbewerberleistungsgesetz ersetzt haben sowie die deutsche Mindestsicherung sich heute zwischen der Scylla einer angestrebten höheren Zivilgenauigkeit (,better targeting“) und besseren Klientenorientierung einerseits und der Charybdis einer politischen Fragmentierung der Mindestsicherungsklientel und -lobby andererseits bewegt.

In Großbritannien setzt Central Government auch die Leistungssätze für die soziale Mindestsicherung fest, die sich tendenziell eher an den Verbraucherpreisen orientieren, und tut dies ohne ausdrückliche Bezugnahme auf empirische Kriterien und spezifische Gerechtigkeitserwägungen.

Die hierzulande in der Vergangenheit unternommenen Versuche, das sozio-kulturelle Existenzminimum wissenschaftlich abzuleiten - dies nicht zuletzt wegen der verfassungsrechtlich vorgegebenen Aufgabe, den Anspruchsberechtigten die Führung eines Lebens zu ermöglichen, das der Würde des Menschen entspricht - findet in Großbritannien (unbeschadet der langen Tradition der Armenforschung) auf politischer und administrativer Ebene keine Entsprechung.

\section{Die Rolle des Rechts und der Gerichtsbarkeit bei der Verwirklichung sozialer Rechte}

„For lawyers and the courts (...) the period from 1970 to 2000 provided a practiced and psychological transformation comparable with the earlier constitutional revolution." 26

„Für den Juristen des europäischen Kontinents ist das englische Recht schon immer etwas besonders Fremdartiges und Wundersames gewesen. "27

Wirft man mit dem Blick auf Großbritannien die Frage nach den Besonderheiten des angelsächsischen Rechts auf, so denkt man aus deutscher Sicht gemeinhin zum einen an das Fehlen einer geschriebenen Verfassung (wobei das Fehlen einer Verfassungsurkunde natürlich nicht das Fehlen einer Verfassung im materiellen Sinne überhaupt indiziert) und zum anderen an das klassische Fallrecht („Case Law“) und seine Methode.

In Bezug auf letzteres hat das Interesse an rechtsvergleichenden Studien zum angelsächsischen Recht in den letzten Jahren spürbar zugenommen, spricht man doch auch

26 Stevens, R., The English Judges. Their Role in the Changing Constitution, Oxford 2005, S. 80.

27 Zweigert/Kötz, Einführung in die Rechtsvergleichung (Fußn. 2), S. 17. 
hierzulande von der zunehmenden Bedeutung von Fallrecht ,auf dem Boden alternder Kodifikationen" 28 und zeigt sich eine auch in anderen Zusammenhängen aufscheinende wachsende Nähe von kontinentaleuropäischem - auch deutschem - und angelsächsischem Recht, zumal auch auf einem so „modernen“ Rechtsgebiet, wie es das Sozialrecht bzw. das law of the welfare state/social welfare law ist.

Für das Verständnis angelsächsischen Rechts bleibt dabei ein historischer Zugang für ein rechtes Verständnis unverzichtbar. Das anglo-amerikanische Recht ist herkömmlicherweise dadurch charakterisiert, dass traditionellerweise der Richter und nicht der Gesetzgeber im Zentrum der Rechtsfindung steht und angesichts des Fehlens eines systematischen Gesetzeswerks das richterliche Urteil jedenfalls in der Vergangenheit Hauptgegenstand der juristischen Analyse und der juristischen Tätigkeit überhaupt gewesen ist. ${ }^{29}$ Dementsprechend gliedert sich die klassische anglo-amerikanische Rechtsquellenlehre - vereinfacht formuliert - in zwei große Bereiche, nämlich zum einen das bereits erwähnte klassische Richterrecht - Case Law -, zum anderen das ständig an Bedeutung zunehmende Gesetzesrecht - Statute Law -.

Das britische Verfassungsrecht zeichnet sich dadurch aus, dass die ihm zugrundeliegende Verfassung ,ungeschrieben“ ist. Allerdings gibt es eine Vielzahl von Gesetzen zu Fragen, die zum materiellen Verfassungsrecht und in anderen Staats- und Verfassungsordnungen i. d. R. auch zum Verfassungsrecht im formellen Sinne gehören würden und welche dann dort im jeweiligen Verfassungsdokument niedergelegt wären. Die aus solchen Gesetzen erwachsenden Verpflichtungen binden die Gerichte, nicht aber das Parlament des United Kingdom of Great Britain and Northern Ireland, welches auch insoweit souverän ist, sodass seine „Allmacht“ als das überragende Verfassungsprinzip des Vereinigten Königreichs überhaupt bezeichnet werden - ein Prinzip, welches aber in zunehmendem Maße durch internationales und insbesondere supranationales Recht, d. h. das Europäische Gemeinschaftsrecht, herausgefordert und auch eingeschränkt wird. 30

An dieser Stelle sei daran erinnert, dass auch in Deutschland das Verhältnis von Verfassungsrecht und Verfassungsgerichtsbarkeit einerseits und Europäischem Gemeinschaftsrecht und Europäischer Gerichtsbarkeit andererseits nicht spannungs- und kon-

28 Vgl. Larenz, K., Methodenlehre der Rechtswissenschaft, 6. Aufl. Berlin 1991; auch Fikentscher, K., Methoden des Rechts in vergleichender Darstellung, Bd. II: Anglo-amerikanischer Rechtskreis, Tübingen 1975, S. $240 \mathrm{ff}$.

$29 \mathrm{Vgl}$. zu weiteren Einzelheiten Zweigert/Kötz, Einführung in die Rechtsvergleichung (Fußn. 2), S. 177 ff.; auch Blumenwitz, D., Einführung in das anglo-amerikanische Recht, 7. Aufl. München 2003; Bernstorff, Graf von, Einführung in das englische Recht, 2. Aufl. München 2000. Zu einem Vergleich zwischen den Gerichtsbarkeiten Englands, der USA und - für Kontinentaleuropa ,stehend" - Frankreich vgl. Abraham, H., The judicial process. An Introduction Analysis of the Courts of the United States, England and France, Oxford u. a. 1998.

30 Vgl. zu dieser Entwicklung etwa Wade, H., Administrative Law, 6. Aufl. Oxford 1988; ferner Cane, P., An Introduction to Administrative Law, 3. Aufl. Oxford 2000; de Smith, St./Brazier, R., Constitutional and Administrative Law, 8. Aufl. Harmondsworth 1998. 
fliktfrei ist. ${ }^{31}$ Für das Vereinigte Königreich ist diese Entwicklung angesichts der herausragenden Bedeutung des Parlamentes allerdings sehr viel dramatischer. ${ }^{32}$

Ursprünglich dem angelsächsischen Rechtsdenken unbekannt, findet sich heute auch in Großbritannien - nicht zuletzt unter dem Einfluss kontinental-europäischen Rechtsdenkens - im nationalen Recht (,municipal law') die Unterscheidung von Privatrecht „Private Law" - und öffentlichem Recht - „Public Law“ -. Allerdings stimmen „Public Law" und kontinentaleuropäisches - auch deutsches - öffentliches Recht ihrer Bedeutung nach nicht überein.

Überdies ist in jüngster Zeit zu beobachten, dass im deutschen Recht etwa die Dichotomie von Privatrecht und öffentlichem Recht in zunehmendem Maße an Eindeutigkeit einbüßt, weil sich der Staat in wachsendem Maße privatrechtlicher Formen bedient, insbesondere auch bei der Wahrnehmung sozialer Aufgaben, und dadurch die Abgrenzung schwieriger wird.

Es ist vor allem mit den Folgen der Industriellen Revolution und dem Aufbau des Wohlfahrtsstaates im auslaufenden 19. und im 20. Jahrhundert einhergehende Wandel der Staatsaufgaben, der zu einer Modernisierung der traditionellen Verwaltungsrechtsdoktrin sowie insbesondere auch zur Intensivierung und Effektivierung der richterlichen Kontrolle beigetragen hat.

In diesem Zusammenhang hat im Laufe des 20. Jahrhunderts die Verwaltungskontrolle durch besondere tribunals an Bedeutung gewonnen. Diese institutionalisierte Form der Rechtsschutzgewährung außerhalb des klassischen court-Systems ist auch eine Folge der Wohlfahrtsstaatsgesetzgebung seit dem 2. Weltkrieg und hat sich seit Ende der 1950er Jahre nicht zuletzt als Reaktion auf das Fehlen einer eigenständigen Verwaltungsgerichtsbarkeit etabliert.

Der Beitritt zur Europäischen Wirtschaftsgemeinschaft Anfang der 1970er Jahre hat zu der wohl tiefgreifendsten je stattgefundenen Rechtsänderung und Rechtsfortentwicklung in Großbritannien geführt. Seither sind die Europäischen Gründungsverträge auch Teil der britischen Rechtsordnung und müssen auch von den britischen Gerichten angewendet werden. Das Europäische Gemeinschaftsrecht geht britischem Recht vor. Der European Communities Act 1972 stellt zudem klar, dass die britischen Gerichte auch die Auslegungsgrundsätze des Europäischen Gerichtshofs befolgen müssen.

Vor diesem rechtlichen Hintergrund haben die Gerichte in Großbritannien in jüngerer Zeit einen corpus von Verwaltungsrechtsregeln geschaffen, der es durchaus mit den kontinentalen Verwaltungsrechtssystemen aufnehmen kann. Besonders bemerkenswert sind hierbei sowohl die Offenheit der angelsächsischen Rechtsordnung gegenüber Ein-

31 Hierzu vgl. jüngst Papier, H.-J., Zum Verhältnis des nationalen Rechts zum Europarecht nach der Rechtsprechung des Bundesverfassungsgerichts, in: Müntefering, F./Becker, U. (Hrsg.), 50 Jahre EU - 50 Jahre Rechtsprechung des Europäischen Gerichtshofs zum Arbeits- und Sozialrecht, Baden-Baden 2008, S. 21 ff.

32 Vgl. dazu jüngst Birkinshaw, P., English Public Law under European Influence, in: Schwarze, J. (Hrsg.), Bestand und Perspektiven des Europäischen Verwaltungsrechts. Rechtsvergleichende Analysen, Baden-Baden 2008, S. 97 ff. m. w. N. 
flüssen des Europäischen Rechts und der Europäischen Gerichtsbarkeit, zumal des Europäischen Gerichtshofs, und auch anderer mitgliedstaatlicher Rechtsordnungen mit längerer Verwaltungsrechtstradition, namentlich Frankreichs, als auch die Flexibilität und Anpassungsfähigkeit, die in dieser Rezeption fremden Rechts zum Ausdruck kommen.

Dies geschieht vor dem Hintergrund von Globalisierung, Internationalisierung (und Europäisierung als ihrer regionalen Ausprägung), die dem Recht genauso ihren Stempel aufdrücken wie dies der gesellschaftliche Wandel tut. 33 Das Europäische Gemeinschaftsrecht stellt insofern für die nationalen Rechtsordnungen der Mitgliedstaaten ein immer dichter werdendes Begegnungsfeld dar, welches in Gestalt des Europäischen Gerichtshofs in Luxemburg auch und mithin ein „gemeinsames Interpretationsforum“34 hat. In diesem Sinne ist der Europäische Gerichtshof seit Zugehörigkeit Großbritanniens zum Europäischen Staatenverbund auch ein Ort der Begegnung von kontinentaleuropäischem (einschließlich natürlich deutschem) und angelsächsischem Recht. Das Europäische Gemeinschaftsrecht wiederum gibt einen Rahmen vor, innerhalb dessen diese Rechtsbegegnung stattfindet. ${ }^{35}$

Der das britische Verfassungsrecht prägenden „Grundnorm“ der „Allmacht“ des Parlaments werden von Grundrechten der Bürger (nach kontinental-europäischem und insbesondere auch deutschem Verfassungsverständnis) prinzipiell keine Grenzen gesetzt. Es gibt auch keine ,übergesetzlichen“ (verfassungsrechtlichen) subjektiven Rechte des einzelnen auf soziale Leistungen etwa im Sinne entsprechend durch Gesetzesrecht zu konkretisierender sozialer Grundrechte. Sozial schützenswerten Positionen wird auch nicht derselbe Rang zuerkannt wie den klassischen Freiheitsrechten („,civil liberties“), weil es ihnen an Bestimmbarkeit, Durchsetzungsfähigkeit und Justiziabilität mangelt. ${ }^{36}$ Es gibt auch keine ausdrücklichen höherrangigen objektiven Verpflichtungen des Staates zur Sozialleistungsgewährung.

Allerdings setzen die „rule of law“ als Ausdruck der Rechtsstaatlichkeit und überkommene, ungeschriebene Wertvorstellungen und Verhaltensregeln der Parlamentsallmacht Grenzen und schreiben auch ein bestimmtes $\mathrm{Maß}$ an Sozialstaatlichkeit, insbesondere die Gewährung eines sozialen Minimums vor. Auch weist die einfachgesetzli-

33 Vgl. zu dieser Entwicklung z. B. Atiyah, P., Law and Modern Society, 2. Aufl. Oxford u. a. 1995; Friedmann, K., Law in a Changing Society, 2. Aufl. Harmondsworth 1972.

34 Vgl. zu diesem Element der Herausbildung einer Europäischen Rechtskultur etwa Häberle, P., Europäische Rechtskultur, Versuch einer Annäherung in zwölf Schritten, Baden-Baden 1994, S. 9 ff.

35 Vgl. exemplarisch dazu Schwarz, K.-A., Vertrauensschutz als Verfassungsprinzip, Eine Analyse des nationalen Rechts, des Gemeinschaftsrechts und der Beziehungen zwischen beiden Rechtsweisen, Baden-Baden 2002.

$36 \mathrm{Zu}$ dieser Grundrechtsproblematik vgl. Loewenstein, K., Staatsrecht und Staatspraxis von Großbritannien, Berlin 1967, Bd. 2, S. 261 ff.; Kingston, P./Imrie, C., Vereinigtes Königreich von Großbritannien und Nordirland, in: Grabitz, E. (Hrsg.), Grundrechte in Europa und USA, Kehl 1986; Pieters, $D$., Sociale Grondrechten op prestaties en de grondwetten van de landen van de Europese Gemeenschap, Antwerpen 1985, S. 459 ff.; auch ders., Soziale Grundrechte in den Mitgliedstaaten der EG, in: von Maydell, B. (Hrsg.); Soziale Rechte in der EG, Berlin 1990, S. 21 ff. 
che Einräumung sozialer Rechte in Großbritannien eine bis ins Mittelalter zurückreichendem „Poor Law“ ihren Niederschlag findende Tradition auf. 37

Die fehlende verfassungsrechtliche Absicherung grundrechtlicher Positionen des Einzelnen wird schließlich in gewissem Umfang durch außerrechtliche, insbesondere politische Begrenzungen ausgeglichen.

In den letzten Jahren hat die Antidiskriminierungsgesetzgebung insbesondere im Hinblick auf die Gleichbehandlung aller EU-Bürger und in Bezug auf die rechtliche Gleichstellung von Männern und Frauen auch im Recht der sozialen Sicherheit - hier vor allem auch unter dem Einfluss und der Geltung einschlägigen Europäischen Gemeinschaftsrechts - primärrechtlich Art. 141 EG (ex 119 EWGV) und sekundärrechtlich die diese Vorschriften umsetzenden Richtlinien - zunehmende Bedeutung erlangt. Vom supranationalen Recht geht insgesamt in Gestalt der sozialen Grundrechten, auch des Grundrechts auf soziale Unterstützung, ein deutlicher "Schub“ aus in Richtung auf ein Mehr an rechtlichen Bestandsschutz für soziale Rechte und Anwartschaften, ${ }^{38}$ nicht zuletzt wegen der Verbürgungen der bis zum Inkrafttreten des an die Stelle des gescheiterten Europäischen Verfassungsvertrags (,Vertrag über eine Verfassung für Europa“) getretenen, aber dessen Essentialia wahrenden Vertrags von Lissabon noch nicht rechtsverbindlichen, aber bereits politisch wie rechtlich vorwirkenden Charta der Grundrechte der Europäischen Union (GRCh) und ihren sozialen Grundrechten:

Art. 34 Abs. 3 GRCh:, (...) anerkennt und achtet die Union das Recht auf eine soziale Unterstützung ..., die allen, die nicht über ausreichende Mittel verfügen, ein menschenwürdiges Dasein sicherstellen sollen, (...)“

Vor allem aus dem Sozialstaatsprinzip (Art. 20 u. 28 GG) als verfassungsrechtlicher Verankerung des sozialen Staatsziels ${ }^{39}$ entspringt die Verpflichtung des deutschen Gesetzgebers, Arbeitslose bei der Sicherung des Lebensunterhalts und der Suche nach einem Arbeitsplatz zu unterstützen. Diese verfassungsrechtliche Verpflichtung trägt dem Umstand Rechnung, dass die Ausübung einer Erwerbstätigkeit nicht nur ermöglicht, den finanziellen Lebensunterhalt für sich und die Seinen zu sichern, sondern auch eine Grundbedingung für die freie Entfaltung der Persönlichkeit und ein Maßstab für die gesellschaftliche Anerkennung ist, die der Einzelne genießt.

Allerdings ist dieser Auftrag an den Gesetzgeber, für Arbeitslose zu sorgen, im Hinblick auf das „Wie“ nicht weiter konkretisiert mit der Folge, dass die Verfassung dem deutschen Gesetzgeber einen weiten Raum für politische Gestaltung gibt. Das Grundge-

37 Siehe dazu bereits oben 2 .

38 Vgl. zu dieser Problematik Schulte, B., Bestandsschutz sozialrechtlicher Positionen, Eine vergleichende Betrachtung, ZIAS 1988, S. 205 ff.; ders., Welche Garantien bieten die Systeme der sozialen Sicherheit?, in: Tomandl , Th. (Hrsg.), 100 Jahre Sozialversicherung in Österreich, Wien 1989.

39 Vgl. zu diesem Begriff und seiner Bedeutung Zacher, H., Das soziale Staatsziel, in: Isensee, J./Kirchhof, P. (Hrsg.), Handbuch des Staatsrechts, Bd. 2: Verfassungsstaat, 3. Aufl. Heidelberg 1995, S. 659 ff. 
setz lässt insofern staatliche Interventionen in den Arbeitsmarkt zu, ${ }^{40}$ verpflichtet aber nicht zu konkreten Maßnahmen.

Allerdings ist in diesem Zusammenhang zu berücksichtigen, dass die Verfassung ihre integrative Kraft für die Gestaltung des Staatswesens nur entfalten kann, wenn die stets vorhandene - Spannung zwischen der normativen Verfassung und der realen Verfasstheit nicht zu groß wird; hieraus mag sich ggf. ein mittelbarer Zugang zu sozialer Intervention ergeben.

In Großbritannien hat der Staat seit jeher weniger stark in den Arbeitsmarkt interveniert, als dies in Deutschland der Fall gewesen ist. Dementsprechend waren Mitte der 1990er Jahre die Aufwendungen für eine ,aktive“ Beschäftigungspolitik - gemessen in Vomhundertsätzen des Bruttoinlandprodukts - auch nur etwa halb so hoch wie hierzulande. Einem gesellschaftlichen Konsens, demzufolge die Rolle des Staates auf dem Arbeitsmarkt möglichst gering sein solle - im Sinne eines liberalen Konzepts von „Labour Relations“ -, entspricht eine verfassungsrechtliche Ausgangslage, die dadurch gekennzeichnet ist, dass es keine richterliche Überprüfung der Verfassungsmäßigkeit von Gesetzen gibt, sodass dem britischen Gesetzgeber im Hinblick auf beschäftigungspolitische Maßnahmen und zumal auch in Bezug auf Leistungseinschränkungen im Bereich der sozialen Sicherheit keine verfassungsrechtlichen Verbürgungen die Hände binden. ${ }^{41}$ Wegen des Fehlens derartiger Schranken war die Durchsetzung deregulierender Maßnahmen auf dem britischen Arbeitsmarkt sehr viel rascher und durchgreifender möglich, als dies in Deutschland angesichts verfassungsrechtlicher „Checks and Balances“ möglich gewesen wäre und unter dem Vorzeichen der Agenda 2010 und von Hartz IV zu Beginn dieses neuen Milleniums möglich gewesen ist.

Insgesamt ist das britische System der sozialen Sicherheit somit sehr viel weniger verrechtlicht (auch im Sinne von „vergesetzlicht“ und „,vergerichtlicht“) als die Systeme der kontinental-europäischen Länder ${ }^{42}$ und auch das deutsche System der sozialen Sicherheit.

40 BVerfGE 50, 290 ff., $336 \mathrm{ff}$.

41 Vgl. in diesem Sinne etwa Danwitz, Th. von, Arbeitsmarkt und staatliche Lenkung, in: Veröffentlichungen der Vereinigung der deutschen Staatsrechtslehre 59 (2000), Berlin u. a., S. 99 ff., 116.

42 Vgl. zu dieser Problematik - insbes. auch im Vergleich zur Situation in der Bundesrepublik Deutschland - Brooke, R., Law, Justice and Social Policy, London 1979, insbes. S. 99 ff.; Williams, $D$., Judicial Restraint and Judicial Review: The Role of the Courts in Welfare Law, in: Partington, M. (Hrsg.) Welfare Law \& Policy, London 1979, S. $101 \mathrm{ff.}$

Zum verfassungs- und verwaltungsrechtlichen Hintergrund vgl. Wade, E./Bradely, A., Constitutional and Administrative Law, London 1985; insbesondere zum Verwaltungsrecht vgl. Foulkes, D., Administrative Law, 6. Aufl. London 1986; Garner, J./Jones, B., Garner's Administrative Law, 6. Aufl. London 1985, insbesondere S. 399 ff.

Zur „Verrechtlichungsdebatte” vgl. umfassend Teubner, G. (Hrsg.), Juridification in Social Spheres, Berlin 1987; zu Großbritannien vgl. Partington, M., ebda.; zu einer Charakterisierung des deutschen Sozialstaats aus britischer Sicht, welche die Besonderheiten beider „Sozialrechtskulturen“ deutlich macht, vgl. Ogus, A., The Federal Republic of Germany as Sozialstaat: A British Perspective, Manchester 1990. 
Sieht man den Unterschied zwischen (britischem) Welfare State und (deutschem) Sozialstaat vor allem darin, dass ersterer Begriff eine beschreibende (vor allem sozialwissenschaftliche), letzterer hingegen eine normative (namentlich rechtswissenschaftliche) Funktion hat und mit spezifischen freiheitsrechtlichen, demokratischen und rechtsstaatlichen Konnotationen versehen ist, so ist Großbritannien in diesem Sinne kein Sozialstaat, hat doch dort die Regierung - wie sich gerade an der Reformpolitik der Konservativen Regierung in den 1980er Jahren aufzeigen lässt ${ }^{43}$ - sehr viel freiere Hand, Veränderungen und insbesondere auch Einschränkungen im Sozialleistungsbereich vorzunehmen, als dies im sozialen Rechtsstaat Bundesrepublik Deutschland der Fall ist. Auch ist hierzulande die sozialpolitische Diskussion sehr viel stärker rechtlich (und insbesondere verfassungsrechtlich) geprägt als jenseits des Kanals, so dass man insofern von einer spezifischen „sozialstaatlichen Rhetorik“, wenn nicht sogar von einer spezifischen „sozialstaatlichen Kultur“" sprechen kann, die (hier aus Raumgründen nicht weiterauszuführende) Vorteile, aber auch Nachteile aufweist. Die Sozialstaatlichkeit führt ferner dazu, dass sowohl rechtlich als auch politisch ein Konsens über das wohlfahrtsstaatlich Erreichte wie das wohlfahrtsstaatlich Erstrebte rechtlich fixiert ist und eine hohe Bindungswirkung entfaltet.

\section{Reform der Arbeitsförderung in Großbritannien und in Deutschland:} „Harrods “ und ,Hartz"

\section{Europarechtliche und -politische Vorgaben}

Seit Inkrafttreten des Vertrags von Amsterdam wird das sowohl in Art. 2 EU-Vertrag (EU) als auch in Art. 2 EG-Vertrag (EG) verankerte Ziel von Europäischer Union und Europäischer Gemeinschaft, ein hohes Beschäftigungsniveau zu fördern, durch den Titel VIII. Beschäftigung (Art. 125 - 130 EG) des EG-Vertrages konkretisiert. Mittel zur Erreichung dieses beschäftigungspolitischen Ziels ist die sog. Europäische Beschäftigungsstrategie (EBS), die auf Ausbildung, berufliche Qualifizierung und Anpassungsfähigkeit der Arbeitnehmer abzielt und von den Mitgliedstaaten als Angelegenheit von gemeinsamem Interesse angesehen wird.

In einem Beschäftigungsausschuss ist diese Zusammenarbeit der Mitgliedstaaten in der Beschäftigungspolitik institutionalisiert und durch die Erstellung von Leitlinien und die Verabschiedung von Schlussfolgerungen sowie Empfehlungen des Rates konkretisiert worden. Die Beschäftigungspolitik ist damit zwar nach wie vor Angelegenheit der Mitgliedstaaten, aber in wachsendem Maße europapolitisch beeinflusst.

43 Siehe dazu oben 3. 
Weitere, hier nur zu erwähnende europarechtlichen Vorgaben gibt es primärrechtlich, d. h. in den Europäischen Gründungsverträgen im Rahmen der - insgesamt sehr begrenzten - Zuständigkeiten der Europäischen Gemeinschaft für die Sozialpolitik gemäß Art. 136 ff. EG - u. a. in Bezug auf Förderung der Zusammenarbeit der Mitgliedstaaten auch im Bereich der beruflichen Eingliederung (Art. 137 EG) und der Beschäftigungsförderung (Art. $140 \mathrm{EG}$ ) -, über die wirtschaftlichen Grundfreiheiten, d. h. namentlich die Personenfreiheiten - Arbeitnehmerfreizügigkeit (Art. 39 EG), Niederlassungsfreiheit Selbständiger (Art. 43 EG) und Dienstleistungsfreiheit (Art. 49 EG) - mit ihren Auswirkungen auch auf die Beschäftigungspolitik und den Sozialschutz, die mit Diskriminierungsverboten wegen der Staatsangehörigkeit eines Mitgliedstaats und allgemeinen Beschränkungsverboten einhergehen, sowie sekundärrechtlich in Gestalt des Europäischen koordinierenden Sozialrechts der Verordnungen (EWG) Nr. 1408/71 und Nr. 574/72 (demnächst Verordnung (EG) Nr. 883/2004 und ihre noch zu erlassende Durchführungsverordnung), der sog. „Freizügigkeitsverordnung“ - Verordnung (EWG) Nr. 1612/68 -, der Richtlinie über Dienstleistungen im Binnenmarkt u. a. Konkretisierungen und Spezifizierungen erfahren haben, sowie in Gestalt der Vorschriften des Europäischen Wettbewerbsrechts mit dem für die Beschäftigungspolitik besonders relevanten Europäischen Beihilfenrecht (Art. 87 EG) und seinem grundsätzlichen Verbot staatlicher Beihilfen, die den zwischenstaatlichen Wettbewerb im Gemeinsamen Markt beeinträchtigen. ${ }^{44}$

\section{Aktivierungspolitik in Großbritannien und Deutschland in Stichworten}

a) Großbritannien: Die konservative Ära und Labour's Third Way

\begin{tabular}{|c|c|}
\hline Leistungssystem & $\begin{array}{l}\text { contribution-based und income-based und } \\
\text { means-tested Jobseeker's Allowance (bei- } \\
\text { tragsbasierte und bedürftigkeitsgeprüfte } \\
\text { Unterstützung für Arbeitsuchende mit } \\
\text { gleichem Leistungsniveau), wie bisher } \\
\text { einheitliche Leistungen } \\
\text { New Deals als Maßnahmen der gezielten } \\
\text { aktiven Arbeitsförderung für einzelne } \\
\text { Gruppen: Jugendliche, Langzeitarbeitslo- } \\
\text { se, Alleinerziehende, Behinderte u. a. }\end{array}$ \\
\hline Organisation & $\begin{array}{l}\text { Department for Work and Pensions (Mi- } \\
\text { nisterium für Arbeit und Renten) und Job- } \\
\text { centres/Zentralisierung }\end{array}$ \\
\hline
\end{tabular}

44 Vgl. Finn/Schulte (Fußn. 1), S. 195 ff. 


\begin{tabular}{|l|l|}
\hline Durchführung & $\begin{array}{l}\text { Jobcentre Plus: Integration von Dienst- } \\
\text { und Geldleistungen }\end{array}$ \\
\hline Finanzierung & Central Government \\
\hline $\begin{array}{l}\text { Flankierende Maßnahmen } \\
\text { („making work pay“) }\end{array}$ & $\begin{array}{l}\text { Minimum Wage (gesetzlicher Mindest- } \\
\text { lohn) und Tax Credits (Steuergutschrif- } \\
\text { ten/Negativsteuern) }\end{array}$ \\
\hline
\end{tabular}

b) Deutschland „Fördern und Fordern“: „Moderne Dienstleistungen am Arbeitsmarkt" als Teil der ,Agenda 2010“"

\begin{tabular}{|l|l|}
\hline Leistungssystem & $\begin{array}{l}\text { Soziale Grundsicherung für Arbeit Su- } \\
\text { chende (erwerbsfähige Personen) }\end{array}$ \\
\hline Leistungsniveau & $\begin{array}{l}\text { Herabsetzung des bisherigen versiche- } \\
\text { rungsrechtlichen Leistungsniveaus und } \\
\text { Sozialhilfeniveaus (Bedürftigkeitsprü- } \\
\text { fung) und Verkürzung der Leistungsdauer }\end{array}$ \\
\hline Organisation & $\begin{array}{l}\text { Bundesagentur für Arbeit und Kommunen } \\
\text { (mit sozialer Selbstverwaltung durch die } \\
\text { Sozialpartner und Mitverantwortung und } \\
\text { Mitwirkung der Kommunen und damit } \\
\text { Dezentralisierung) }\end{array}$ \\
\hline Finanzierung & Bund \\
\hline Durchführung & $\begin{array}{l}\text { Jobcenter - Zusammenarbeit von Bundes- } \\
\text { agentur für Arbeit und Kommunen }\end{array}$ \\
\hline Flankierende Maßnahmen & $\begin{array}{l}\text { „Ich-AGs“, „Mini-Jobs“; Einbeziehung } \\
\text { erwerbsfähiger Hilfsbedürftiger in die } \\
\text { Kranken-, Pflege- und Rentenversiche- } \\
\text { rung }\end{array}$ \\
\hline $\begin{array}{l}\text { Anspruchsvoraussetzungen für Förde- } \\
\text { rungsleistungen }\end{array}$ & $\begin{array}{l}\text { Arbeitslosigkeit statt bloßer Verfügbarkeit } \\
\text { auch Verpflichtung zu aktiver Arbeitssu- } \\
\text { che sowie Eingliederungsvereinbarung: } \\
\text { Verpflichtung des Einzelnen zur Arbeits- } \\
\text { suche und des Staates zur Unterstützung } \\
\text { dieser Bemühung }\end{array}$ \\
\hline
\end{tabular}




\section{c) Würdigung}

Wenn sich auch seit Geltung der Europäischen Menschenrechtskonvention und unter Einfluss des Rechts der Europäischen Union gleichsam „von Europa her“ ein Wandel in Richtung auf „mehr Recht“ abzeichnet, bleibt gleichwohl „unter dem Strich“ festzuhalten, dass Arbeitsrecht und Sozialrecht im Vereinigten Königreich sehr viel flexibler gehandhabt werden können und auch gehandhabt werden, als dies im deutschen zumal bundesstaatlich verfassten sozialen Rechtsstaat der Fall ist.

So konnten beispielsweise in Großbritannien die erwähnten Zuschläge zu den kurzzeitigen Einkommensersatzleistungen $u$. a. auch bei Arbeitslosigkeit abgeschafft werden, ohne dass sich die von dieser Maßnahme Betroffenen etwa wirksam auf einen rechtlichen Vertrauensschutz als Ausfluss des Rechtsstaatprinzips - in Großbritannien der „rule of law“ - oder gar auf einen grundrechtlichen Eigentumsschutz sozialer Anwartschaften hätten berufen können.

\section{Großbritannien und Deutschland - Gemeinsamkeiten und Unterschie- de in Stichworten}

Die maßgeblichen Reformen fanden im Vereinigten Königreich seit Mitte der 1980er Jahre, in Deutschland seit 1998 (SGB III) statt.

Beteiligte Leistungssysteme sind im Vereinigten Königreich die Jobseeker's Allowance, i. e. Verschmelzung („Fusion“) von Unemployment Benefit (Arbeitslosengeld) und Income Support (Einkommensunterstützung) für Erwerbsfähige, in Deutschland die Zusammenlegung von Arbeitslosen und Sozialhilfe in Gestalt von Arbeitslosengeld II, Grundsicherung für Arbeitsuchende (statt bisher Arbeitslosenhilfe und Sozialhilfe für erwerbsfähige Personen).

Die Organisation erfolgt in Großbritannien zentralstaatlich (ohne Beteiligung von Kommunen und Sozialpartnern), in Deutschland durch die Bundesagentur für Arbeit und Kommunen unter Beteiligung der Sozialpartner.

Leistungsmodalitäten sind in beiden Ländern die Verpflichtung zur aktiven Arbeitsuche, die Verschärfung der Zumutbarkeitskriterien und die Absenkung des Leistungsniveaus zur Erhöhung des Anreizes zur Aufnahme einer Erwerbstätigkeit.

Großbritannien kennt mit dem Jobseeker's Agreement eine Vereinbarung über die Arbeitsuche sowie in Gestalt der New Deals spezifische Förderungsmaßnahmen für einzelne Gruppen (Alleinerziehende, Berufsanfänger, Langzeitarbeitslose, Behinderte) und Personal Adviser als individuelle Anlaufstelle (hohe Kontaktdichte und Betreuungsrelation). Auch Deutschland kennt eine Eingliederungsvereinbarung. 
Leistungen zum Lebensunterhalt sind in Großbritannien die Jobseeker's Allowance (Unterhaltsgeld für Arbeitssuchende) und in Deutschland die soziale Grundsicherung für Arbeitsuchende.

Weitere Maßnahmen sind „Fördern“/„Enabling“ der Beschäftigungssuche, Beschäftigungsförderung durch Berufsberatung, Arbeitsvermittlung und Hilfe bei der Suche nach einem Arbeitsplatz, Arbeitsförderungsmaßnahmen/Schulung/Berufs-bildung/Umschulung, Finanzielle Hilfen, Arbeitsplatzzuschüsse, Umzugskostenerstattung, Erstattung von Bewerbungskosten, Steuerliche Erleichterungen (,make work pay“) und gesetzlicher Mindestlohn, Freibeträge für Erwerbseinkommen und Lohnzuschüsse, soziale Dienstleistungen wie case management, psychologische und soziale Beratung und Hilfe, Kinderbetreuung.

„Fordern“/,Demanding“ von Arbeitsuchenden geschieht durch Absenkung der Leistungen auf Sozialhilfeniveau, Reduzierung der Leistungsbezugsdauer, strengere Verfügbarkeitskriterien und Verpflichtung zur Arbeitsuche, intensive persönliche Bemühungen um einen Arbeitsplatz sowie durch die Vereinbarung über die Arbeitsuche, die Kontrolle der Bemühungen zur Arbeitssuche, scharfe Sanktionen bei fehlender oder mangelhafter Suche nach Arbeit oder bei Ablehnung einer Arbeitsgelegenheit und Weigerung der Teilnahme an Arbeitsgelegenheiten und Beschäftigungsmaßnahmen.

\section{Resümee}

In Vorhandensein und Ausmaß rechtlicher Gewährleistungen und der entsprechenden gerichtlichen Durchsetzungsmöglichkeiten für die beteiligten Akteure des Arbeitsmarktes, zumal die Arbeitnehmer, liegt ein wesentlicher Unterschied zwischen Deutschland und dem Vereinigten Königreich.

Das Urteil des Bundesverfassungsgerichts zur Verfassungswidrigkeit des Modells der Arbeitsgemeinschaften von Bundesagentur für Arbeit und Kommunen wegen unzureichender Regelung der jeweiligen Verantwortlichkeiten ${ }^{45}$ sowie die Diskussion um Menschenwürde (Art. $1 \mathrm{GG}$ ) und Sozialstaatsprinzip (Art. 20 u. $28 \mathrm{GG}$ ) als verfassungsrechtliche Maßstäbe und Vorgaben für die als soziale Grundsicherung und Sozialhilfe gewährten Leistungen ( $§ 1$ Abs. 1 SGB XII i. V. m. Art. 1 S. 1 GG) und ggf. für das Verfahren zur Ermittlung und Bestimmung des sozio-konventionellen Existenzminimums) sowie die Rechtsqualität der Eingliederungsvereinbarung mit dem Arbeitsuchenden, Sanktionen und gerichtlicher Kontrolle fallen in beiden Ländern unterschiedlich aus.

Ergebnis ist eine divergente Konvergenz, d. h. eine Konvergenz der Ziele und - partiell - auch der Instrumente - so im Hinblick auf monetäre Unterstützungsleistungen der Beschäftigungspolitiken beider Länder.

45 BVerfG, Urt. v. 20.12.2007 (2 BvR 2433, 2434/04), NZS 2008, S. 198 ff. 
Unterschiede bestehen hingegen im Hinblick auf Grad der Zentralisierung, Beteiligung von Regionen und Kommunen sowie der Sozialpartner.

Graduelle Unterschiede bestehen auch im Hinblick auf die Stärkung der Eigenverantwortlichkeit und die „Neuaustarierung“ der sozialen Gerechtigkeit von der Bedarfszur Leistungsgerechtigkeit und in Bezug auf die „Regression“ der Solidarität.

Die Abstimmung der Aktivierungspolitiken beider Länder erfolgt im Rahmen der Europäischen Beschäftigungsstrategie (EBS), die zugleich als eine wichtige Komponente des Europäischen Sozialmodells fungiert.

Die Alliteration „Harrods“ und „Hartz“46 bringt zum Ausdruck, dass sich die entsprechenden Politiken sehr ähneln: Von der Zielsetzung her - „Activation“/,Aktivierung“ -, von der Semantik her - „Jobcentre“ und „Jobseeker“ bzw. ,Jobcenter“ -, während die Umsetzung nach wie vor unterschiedlich, weil „pfadabhängig“ ist (wofür in der politikwissenschaftlichen Literatur der Begriff der „divergenten Konvergenz" geprägt worden ist).

In der Zusammenlegung von Unemployment Benefit und Income Support zu einem einheitlichen Leistungssystem der Jobseeker's Allowance sowie von Arbeitslosenhilfe und Sozialhilfe für Erwerbsfähige ist allerdings auch instrumentell eine Annäherung beider Systeme erfolgt, die im Hinblick auf die monetäre Leistungsseite besonders ausgeprägt ist: Hier hat in Deutschland eine Orientierung weg von der Lebensstandardsicherung hin zu einer bloßen Grundsicherung stattgefunden, wie sie im Vereinigten Königreich seit jeher üblich ist, während die Arbeitsförderungsmaßnahmen hierzulande bei Bundesagentur und Kommunen angesiedelt sind.

Die organisatorischen und administrativ-instrumentellen Unterschiede lassen sich in den Gegensatzpaaren (britische) Zentralisierung versus (deutsche) Bundesstaatlichkeit und dem Kommunalverfassungsrecht plus Elementen sozialer Selbstverwaltung durch die Sozialpartner und einer in Großbritannien so nicht anzutreffenden Freien Wohlfahrtspflege geschuldete Dezentralisierung, politisch-aktionistischem „Enabling Welfare State“ versus ,verfassungsrechtlich gebundener Sozialstaatlichkeit“, sowie - im Hinblick auf den Bürger - „Enabling and Demanding“ versus „Fördern und Fordern“, wobei dank des höheren Grades der Verrechtlichung und „Vergerichtlichung“ das Verhältnis zwischen Arbeitsuchendem und Staat hierzulande im Hinblick auf die wechselseitigen Rechte und Pflichten ausweislich der Eingliederungsvereinbarung sowie der $\mathrm{Zu}$ mutbarkeitskriterien und Verpflichtungen zur Arbeitsuche, Sanktionen und ihrer gerichtlichen Kontrolle stärker ausgestaltet und kontrolliert ist.

Dabei ist im britischen „Employment First“ Welfare State das Zusammenwirken zwischen Arbeitsverwaltung und Arbeitsuchenden intensiver, z. B. ausweislich der Rolle des Personal Adviser als Case-Manager, der Dichte des Netzes der Jobcenter und der daraus resultierenden Kontaktdichte zwischen Behörde und Klienten und einer besseren Betreuungsrelation (wobei allerdings Großbritannien bereits auf fast zwei Jahrzehnte,

46 Vgl. zu dieser farbigen Terminologie Knuth, M./Finn, D., Hartz oder Harrods?, Reformen der Arbeitsförderung im Vereinigten Königreich, IAT (= Institut Arbeit und Technik) Report 2004-04. 
Deutschland aber erst auf 10 Jahre Aktivierungspolitik am Arbeitsmarkt zurückblicken kann und die deutschen Ergebnisse und Erfahrungen dementsprechend insgesamt weniger - auch weniger gut evaluiert und publiziert - sind). ${ }^{47}$

Demgegenüber beschränkt sich das Recht in Großbritannien stärker darauf, sozialpolitische Entwicklungen widerzuspiegeln, als sie aktiv zu gestalten (dementsprechend ist der sozialpolitische Diskurs in Großbritannien auch weniger rechtlich und insbesondere verfassungsrechtlich geprägt, als dies in Deutschland der Fall ist).

Zugleich nehmen politische Garanten dort in stärkerem Umfang Funktionen wahr, die hierzulande dem Recht zugewiesen sind.

Dies führt z. B. dazu, dass der Bestandsschutz sozialer Rechte und Anwartschaften in Deutschland durch das Recht - etwa aufgrund der Rechtsprechung des Bundesverfassungsgerichts im Hinblick auf beitragsfinanzierte Leistungen, namentlich Altersrenten über Art. 14 GG durch das Grundrecht auf Eigentum und in Ansehung des sozialen Existenzminimums über Art. 1 GG durch den Grundsatz der Menschenwürde - in sehr viel höherem Maße rechtlich gewährleistet wird, als dies durch Recht und Politik im Vereinigten Königreich der Fall ist.

Einen Beleg dafür liefert der vorstehend angesprochene Umbau des britischen Wohlfahrtsstaats von Ende der 1970er Jahre, der auch vor Schritten nicht Halt gemacht hat, welche hierzulande aus verfassungsrechtlichen Gründen kaum durchführbar gewesen wären, wie z. B. der Abschaffung der beitragsfinanzierten und einkommensbezogenen deswegen in Deutschland als Anwartschaften der Versicherten in ihrem Bestand als Eigentum ${ }^{48}$ geschützten - Einkommensersatzleistungen bei Krankheit in Gestalt von sickness benefit (Krankengeld).

An dieser Stelle tritt der Unterschied zwischen dem sozialen Rechtsstaat deutscher Provenienz und dem britischen Wohlfahrtsstaat deutlich zutage. 49

Ansonsten lässt sich aber in der britischen und deutschen Arbeitsmarktpolitik eine schleichende Konvergenz/creeping convergence beobachten, die ausgetretene sozialpolitische Pfade verlässt und einen Paradigmenwechsel anzeigt - auf deutscher Seite noch stärker als jenseits des Kanals. ${ }^{50}$

Man mag in dieser auch auf die gemeinschaftliche Europäische Politik zurückführende Entwicklung 51 auch ein Versatzstück des Europäischen Sozialmodells erblicken, welches bei aller seiner Unbestimmtheit zumal beim Blick ,von außen“, d. h. von Drittstaaten her durchaus existent ist.

47 Vgl. dazu Finn/Schulte (Fußn. 1), S. 297 ff.

48 Vgl. hierzu die umfangreiche Judikatur des Bundesverfassungsgerichts zum Eigentumsschutz sozialversicherungsrechtlicher Ansprüche und Anwartschaften: BVerfGE 53, 257 ff.; 72, 9 ff.; 92, 365 ff. u. a.

$49 \mathrm{Zu}$ diesem deutsch-britischen Vergleich unter juristischem Blickwinkel siehe oben 5.

50 Vgl. Mohr. K., Creeping convergence - Wandel der Arbeitsmarktpolitik in Großbritannien und in Deutschland, ZSR 2008, S. 187 ff. m. w. N.

51 Siehe dazu oben 6.1 . 
Zacher hat auf der Jahrestagung 2005 des Forschungsnetzwerks Alterssicherung dieses Europäische Sozialmodell - vielleicht allzu reduktionistisch - apostrophiert als ,Zunächst offenbar nur so etwas wie eine Ausschreibung: Wer hat die zündende Formel, auf die sich so gut wie alle einigen können?" 52 Die nationalen Aktivierungspolitiken der EU-Staaten beispielsweise - hierfür „stehen“ Großbritannien und Deutschland partes pro toto - haben bei allen ihren nationalen Besonderheiten nicht allein beschäftigungspolitische Zielsetzungen, die den Mitgliedstaaten gemeinsam sind, sondern es gibt auch organisatorische und instrumentelle Gemeinsamkeiten.

Das Europäische Sozialmodell ist last but not least der sozialen Gerechtigkeit verpflichtet, deren Komponenten - Bedarfsgerechtigkeit, Leistungsgerechtigkeit, Besitzstandsgerechtigkeit u. a. - durch die Aktivierungspolitik neu gewichtet (und dabei angelegentlich etwas ,aus dem Lot gebracht") worden sind.

Hier stellt sich die Frage, ob nicht die Bedarfsgerechtigkeit und der Grundwert Solidarität zugunsten der Leistungsgerechtigkeit allzu sehr zurückgedrängt worden sind.

In beiden Vergleichsländern erscheint insofern eine „Neutarierung“ der unterschiedlichen und vielschichtigen Komponenten des Konstrukts soziale Gerechtigkeit notwendig.

52 Vgl. Zacher, H., Das „Soziale“ als Begriff des deutschen und des europäischen Rechts, in: Deutsche Rentenversicherung Bund (Hrsg.), Das Soziale in der Alterssicherung (Jahrestagung 2005 des Forschungsnetzwerks Alterssicherung (FNA), Berlin 2006, S. 11 ff. 


\section{Diskussionsbericht}

I. Im Recht der Grundsicherung für Arbeitssuchende dient die Eingliederungsvereinbarung der inhaltlichen Ausgestaltung von Sozialrechtsverhältnissen, indem sie die Leistungen festlegt, die der erwerbsfähige Hilfebedürftige zur Eingliederung in Arbeit erhält, und die aktiven Eingliederungsbemühungen des erwerbsfähigen Hilfebedürftigen konkretisiert.

Zu Beginn der Diskussion im Anschluß an die Beiträge von Bieback, Igl und Schulte zum Themenblock ,Das Leistungsverhältnis in Förderungs- und Hilfesystemen' wurde die elementare Frage aufgeworfen, ob die Eingliederungsvereinbarung im deutschen Recht der Rechtskategorie Vertrag zugeordnet werden könne, wenn bedacht werde, daß der erwerbsfähige Hilfebedürftige als Vertragspartner Objekt eines Fall-Managements sei.

Als Argumente gegen die Vertragseigenschaft der Eingliederungsvereinbarung wurden die fehlende Inhalts- und Abschlußfreiheit sowie die Sanktionsandrohung im Falle des Scheiterns der Eingliederungsvereinbarung vorgetragen. Die Vertragskategorie müsse daher aufgegeben werden, weil die Inhalts- und Abschlußfreiheit, die im Zivilrecht zu den wesentlichen Voraussetzungen eines Vertrages zählten, nicht gegeben seien. Dennoch sei die Eingliederungsvereinbarung sinnvoll. Diese habe zum Gegenstand, die gesetzlich vorgegebenen Ziele möglichst in einem dialogischen Prozeß rational und im gegenseitigen Einvernehmen zu steuern.

Der fehlenden Inhalts- und Abschlußfreiheit als Argument gegen die Vertragseigenschaft der Eingliederungsvereinbarung wurde mit Hinweis auf den subordinationsrechtlichen öffentlich-rechtlichen Vertrag im öffentlichen Recht eine geringe Bedeutung beigemessen. Besonders hervorgehoben wurde in diesem Zusammenhang, daß mit der Sozialhilfe ein intensiverer Zugriff auf die persönliche Lebensführung mit dem Ziel der Steuerung erfolge, dies aber nur begrenzt hoheitlich möglich sei. Zur kooperativen Gestaltung von Sozialrechtsverhältnissen sei das Vertragsgeschehen daher tauglich, auch wenn zwischen den Vertragsparteien Asymmetrien bestünden. Im Rahmen der vertraglichen Gestaltung von Sozialrechtsverhältnissen, in welchen der Staat sehr stark auf das Individuum zugreife, seien aber bestimmte Anforderungen zu erfüllen. Eine zu befürwortende Regelung sei es beispielsweise, wie im französischen und britischen System die Verhängung der angedrohten Sanktionen innerhalb der Vertragsverhältnisse nicht der Zuständigkeit des Vertragspartners zuzuweisen.

Des Weiteren wurde darauf hingewiesen, daß im Rahmen von Sozialrechtsverhältnissen im Unterschied zu den besonderen Gewaltverhältnissen nicht von einem Eindringen in eine Sphäre oder Unterwerfung des Einzelnen gesprochen werden könne; vielmehr würden Abhängigkeiten geschaffen, Freiheiten und Privatsphäre aufgezehrt. Zur Begrenzung der geschaffenen Abhängigkeiten sei es erforderlich, die gegenseitigen Rechte und Pflichten sowie die Ziele und Zielerreichung zumindest in einem Mindest- 